This is a post-peer-review, pre-copyedit version of an article published in the Journal of Signal Processing Systems. The final authenticated version is available online at: https://doi.org/10.1007/s11265-018-1351-1

This version is subjected to Springer Nature terms for reuse that can be found at: https://www.springer.com/gp/openaccess/authors-rights/aam-terms-v1 


\title{
StreamDrive: A Dynamic Dataflow Framework For Clustered Embedded Architectures
}

\author{
Arthur Stoutchinin • Luca Benini
}

the date of receipt and acceptance should be inserted later

\begin{abstract}
In this paper, we present StreamDrive, a dynamic dataflow framework for programming clustered embedded multicore architectures. StreamDrive simplifies development of dynamic dataflow applications starting from sequential reference $\mathrm{C}$ code and allows seamless handling of heterogeneous and applicationspecific processing elements by applications. We address issues of efficient implementation of the dynamic dataflow runtime system in the context of constrained embedded environments, which have not been sufficiently addressed by previous research. We conducted a detailed performance evaluation of the StreamDrive implementation on our Application Specific MultiProcessor (ASMP) cluster using the Oriented FAST and Rotated BRIEF (ORB) algorithm typical of image processing domain. We have used the proposed incremental development flow for the transformation of the ORB original reference $\mathrm{C}$ code into an optimized dynamic dataflow implementation. Our implementation has less than $10 \%$ parallelization overhead, near-linear speedup when the number of processors increases from 1 to 8 , and achieves the performance of 15 VGA frames per
\end{abstract}

Arthur Stoutchinin

ST Microelectronics,

Grenoble France

Tel.: +33-4-76586276

ORCiD: 0000-0002-7650-9570

E-mail: arthur.stoutchinin@st.com

Luca Benini

Electrical, Electronic, and Information Engineering Department,

University of Bologna, Italy,

and

Integrated Systems Laboratory,

Swiss Federal Institute of Technology (ETH), Zurich

ORCiD: 0000-0001-8068-3806

E-mail: luca.benini@unibo.it second with a small cluster configuration of 4 processing elements and $64 \mathrm{~KB}$ of shared memory, and of 30 VGA frames per second with 8 processors and $128 \mathrm{~KB}$ of shared memory.

Keywords embedded, multicore, shared memory, dataflow, kahn process, heterogeneous, accelerator

\section{Introduction}

Advanced embedded computing platforms are often designed as clustered multi-cores $[33,43,36,10]$. In a clustered architecture, processing elements are grouped in tightly-coupled clusters sharing a finite amount of resources such as local memory, a DMA, external access ports, etc. The main disadvantage of such platforms is that software engineers must explicitly deal with parallelism, with heterogeneous and application-specific computing elements, with limited in-cluster memory constraints, and with data transfers across the memory hierarchy. In this paper, we address the programming issues with clustered multi-core platforms.

The dataflow computing model aims at addressing the aforementioned programming challenges for embedded applications that exhibit streaming behavior, eg. image and video processing, multimedia, networking, etc. However, adoption of the dataflow programming model by industry has been hindered by two important issues: (i) the necessity to drastically modify the existing sequential software and software development flow, and (ii) an unappealing trade-off between model expressiveness and efficient implementation. Indeed, restricted dataflow models, such as the Synchronous Dataflow Model (SDF) [30], the Cyclo Static Dataflow Model [5], or the Parameterized Synchronous Dataflow Model [3] are amenable to analysis, 
automation techniques, and efficient implementation, but are too constrained in expressiveness to meet the needs of many real-time industrial applications. On the other hand, dynamic dataflow models, such as Kahn Process Networks (KPN) [27], Boolean Dataflow [26] and Dynamic Dataflow (DDN) [6] are difficult to develop and often do not result in an efficient implementation [19, 50, 34].

In a preliminary publication [55], we presented the StreamDrive framework that supports parallelization of streaming applications and aims at reducing the effort required in doing this. StreamDrive supports two execution modes: preemptive for KPN execution, and cooperative for DDN execution. Based on the simultaneous support for these two execution modes, we propose an incremental transformation flow starting from a sequential reference application and moving towards an optimized dynamic dataflow implementation. Supporting the two execution modes simultaneously is essential for providing such incremental transformation flow because the initial transformation of a sequential algorithm into a KPN often requires minimal modification of the original code. Moreover, the process of transforming the KPN into a DDN by adding dataflow firing rules is relatively straightforward. Another benefit of this methodology is that application-specific hardware blocks, acting as KPN processes, can be seamlessly integrated together with software DDN actors at the application level.

The StreamDrive application programming interface (API) is built on top of the $\mathrm{C}$ language and relies on familiar standard development tools, and the resulting parallel code is not radically different from the initial reference software. Although several dataflow APIs have been proposed in the past (see section 2), none simultaneously combines the support for the KPN and DDN execution modes, while relying on standard $\mathrm{C}$ development tools without introducing language restrictions, and remains sufficiently lightweight for targeting constrained embedded platforms. The StreamDrive communication API allows actors to share the dataflow buffers and efficiently supports data-parallel actors. Finally, the StreamDrive provides a lightweight runtime execution environment where particular attention is paid to minimize the overhead for the run-time support in terms of both, execution cycles and memory footprint requirements. Specific challenges addressed in our work include a low-overhead scheduler, dealing with small memories and the memory hierarchy that needs to be explicitly managed by software. Generally, these issues are insufficiently addressed in existing run-time environments and embedded real-time operating systems.
In [55], we also presented a detailed performance analysis of the dynamic dataflow execution model using a real-life application in a context of a small-scale embedded platform. We have implemented StreamDrive on the embedded Application Specific MultiProcessor (ASMP) platform from ST Microelectronics [52]. We present the results of the evaluation carried out over the Oriented FAST and Rotated BRIEF (ORB) application use cases, which are commonly used in mobile and automotive camera image processing pipelines [49]. Our evaluation showed that the StreamDrive-based ORB implementation achieves real-time performance, low parallelization overhead, small memory footprint, scales near linearly from 1 to 8 processing cores, and maintains performance even with long external memory latency and limited available bandwidth. Compared to other reported publications, our runtime implementation has lower overhead, and our speedup is closer to linear due to efficient combination of two types of parallelism: functional and data parallelism.

This article extends the preliminary version [55] in the following ways. First, we explain in details the StreamDrive communication protocol. Second, we provide a detailed description of the incremental transformation flow starting from the reference code and ending with optimized dataflow implementation, using the ORB as a running example. We explain the details of all the key aspects of the proposed methodology. Finally, we quantify and analyze the performance improvement of a dataflow execution with respect to the KPN execution, and demonstrate that the KPN scheduling overhead is relatively important in a typical embedded multiprocessor context.

The article is organized as follows: related work is explained in section 2 ; in section 3 we give the overview of the ASMP platform, explain its shared memory architecture and its support for the application-specific hardware; in section 4 we present the StreamDrive API and its implementation choices, and we illustrate the StreamDrive incremental transformation flow with an example; finally, we discuss our evaluation results in section 5 .

\section{Related Work}

The dataflow execution model is a popular research topic in the embedded domain because it is a good match for many applications and hardware platforms. Several approaches of dataflow programming have been proposed with the objective to balance conflicting concerns of expressiveness, analyzability, and implementability [54]. Table 1 summarizes selected representative related dataflow publications. 


\begin{tabular}{|c|c|c|c|}
\hline Framework & Model & Target & Programming \\
\hline Ptholemy II & Most existing dataflow models & $\begin{array}{c}\text { Simulation and } \\
\text { design environment }\end{array}$ & Specialized Language \\
\hline LWDF & CFDF & Modeling Framework & C API \\
\hline StreamIT & SDF & Software development & StreamIT Language \\
\hline Sesame & KPN & $\begin{array}{c}\text { Simulation and } \\
\text { design environment }\end{array}$ & C API \\
\hline Kaapi & Dynamic dataflow & $\mathrm{HPC}$ & C API \\
\hline TideFlow & Dynamic dataflow & HPC & C API \\
\hline OpenStream & Control driven dataflow & HPC & OpenMP Extension \\
\hline YAPI & KPN & Workstation & C API \\
\hline Nornir & KPN & Workstation & C API \\
\hline PREESM & PiSDF & $\begin{array}{l}\text { Simulation and code } \\
\text { generation embedded }\end{array}$ & Graphical GUI + C \\
\hline Kalray MPPA & $\mathrm{CSDF}$ & Embedded & $\Sigma C$ Language \\
\hline CAL & SDF,CSDF, Dynamic dataflow & Embedded & Specialized Language \\
\hline $\begin{array}{l}\text { Shim } \\
\text { DOL }\end{array}$ & $\begin{array}{c}\text { Restricted KPN } \\
\text { KPN }\end{array}$ & $\begin{array}{l}\text { Embedded } \\
\text { Embedded }\end{array}$ & $\begin{array}{l}\text { C Extension } \\
\text { C Restricted }\end{array}$ \\
\hline
\end{tabular}

Table 1 Selected related work summary

A large number of frameworks propose specialized languages and tools for developing dataflow applications (the reader is referred to [4] for a comprehensive survey). The premise of these frameworks is that an application can be specified at a high abstraction level and automatically transformed into an efficient implementation. However, in practice there has been a noticeable gap between a high-level description and the efficient implementation that the automated tools failed to close. As a result, often restricted dataflow models are used such as Synchronous Dataflow (SDF) [30], Cyclo Static Dataflow [5], Parameterized SDF [3], Heterochronous Dataflow [20], etc., while achieving the efficiency with more expressive Kahn Process Networks [27], or Dynamic Dataflow [26] remains difficult [19, 50, 34]. Another major inconvenience of these frameworks is that they requires significant changes to reference software and to the existing software development flow reference applications are typically specified as sequential programs using imperative programming languages such as $\mathrm{C} / \mathrm{C}++$ or Matlab. The disruptive changes in software development flow and being able to only deliver efficient implementation for a restricted set of the dataflow models hinders adoption of these technologies by industry.

An alternative is to integrate coarse grain dynamic dataflow programming structures into familiar languages, using a lightweight API with an associated runtime environment. Several such KPN and dynamic dataflow APIs have been proposed in the literature. Many of them target large computing systems and often rely on off-the-shelf OSes. Kaapi [17], Sesame [40], Shim [12] are based on POSIX threads. The QUARK (QUeing And Runtime for Kernels) [61], TIDeFlow [38], and OpenStream [44], have been developed in the context of the High-Performance Computing (HPC) applications. YAPI [28] and Nornir [60] support the KPN execution model on workstation computers. These runtime environments come with heavy performance and memory footprint overheads. This is an acceptable choice for running applications in big-size computers. In the embedded domain we need a lightweight approach: the small memory and the high performance requirements preclude using the full OS, a kernel-level scheduler, and dynamic data structures.

One example of a minimalist dataflow API similar to StreamDrive is the lightweight dataflow (LWDF) [53]. The LWDF implements the core functional dataflow (CFDF) model [41]. In the CFDF, an actor has a set of valid modes in which it can execute. The actor specification is divided into separate enable and invoke functions. The enable is designed to be used as a "hook" for the dynamic scheduler to rapidly query actors at runtime, and check whether or not they are executable. The invoke function implements actor functionality and can generally change the mode of the actor for the next invocation. This is similar to the StreamDrive, where actors proceed deterministically to some "next mode" of execution while changing their firing rules. Plishker et al. [42] have presented an analysis method that can exploit the core functional dataflow to improve the scheduler. The LWDF focus is on providing a framework for modeling and exploring the scheduler strategies, and it does not address the implementation efficiency issues of dynamic dataflow applications.

Several publications addressed supporting static and quasi-static dataflow execution model on embedded platforms. For example, the Parallel and Real-time Embedded Executives Scheduling Method (PREESM) is a framework offering rapid prototyping and automatic 
code generation for heterogeneous multi-core embedded systems. PREESM targets the TI's Keystone DSP architecture and supports the PiSDF model [39]. Another example is the Kalray MPPA system programmed using the specialized $\Sigma \mathrm{C}$ language [21] and implementing the Cyclo Static Dataflow [5].

In order to overcome the limitations of the static dataflow, the Scenario-Aware Dataflow (SADF) [56] views applications as collections of different SDF graphs. SADF is able to perform some worst-case and stochastic analyses, and to provide implementation with limited run-time overhead, while relaxing some of the limitations of the SDF. However, this approach is still limited to a range of applications that follow a sequence of fairly static scenarios. From a syntactic perspective, the SADF model resembles the Heterochronous Dataflow [20], and therefore requires complete re-write of application reference code and usage of specialized development tools.

The above approaches are different from the StreamDrive in that they restrict the computation model to a subclass of dataflow process networks and rely on static scheduling for achieving efficiency.

In order to support dynamic dataflow execution, several research leverage on CAL programming language $[14,15]$, and its ISO-standardized subset, RVCCAL $[31,32]$. The RVC-CAL provides a dataflow framework with high level of abstraction and modularity as a basis for platform independent description of dataflow programs for execution on multicore platforms.

In $[18,58]$, the Actor Machine is used to generate an application-specific runtime dataflow scheduler from CAL targeting the Epiphany architecture [37]. The generated scheduler is less efficient compared to the StreamDrive because the actor machine does not memorize actors blocking conditions and therefore reevaluates these blocking conditions multiple times, while explicit enumeration of actor states leaves large memory footprint. The communication library is tailored to Epiphany's distributed shared memory and does not support sharing of communication buffers. Finally, work in [18] does not support the dynamic mapping of actors on processing elements.

Yviquel et al $[62,63]$ use the RVC-CAL infrastructure for developing a dynamic dataflow framework targeting a shared memory multi-core platform. The framework pays particular attention to the efficient implementation of dataflow communication functions. In particular, the shared memory is used to implement the zero-copy communication channels. The essential difference from StreamDrive lies in how the race conditions are avoided: while StreamDrive defines a communication protocol that ensures the race-free execu- tion, the work in [63] guarantees the atomicity of the sequence read-input/execute/write-output by postponing the update of dataflow channels state until the actor has finished execution. Apart from potential performance overhead, this would be incompatible with the KPN mode of execution. This framework also supports broadcasting single data to several target actors (if all concerned FIFO channels are mapped to the same shared memory bank) that reminds the StreamDrive broadcast operation. However, there is no equivalent to the collect operation and no support for the data parallelism. Finally, the work in [63] relies on static scheduling of actors to platform processing elements.

The RVC-CAL offers a standardized framework for developing dynamic dataflow applications. However, there are very few applications available in CAL, mostly video codecs, while the majority of new applications continue to be developed in standard languages. Thus, the most important difficulty of using CAL is that it requires a complete re-write of the reference applications. CAL tools are also less mature that standard development tools such as the gcc compiler, etc.

Shim $[12,13,59]$ and the Distributed Operation Layer (DOL) [23, 22] addressed implementation issues of the KPN execution model in the embedded context. Shim implements a KPN restricted to support only synchronous (rendezvous) communication. This choice eases scheduling, and programs are, by definition, always executable in finite space because synchronous communication does not need buffering. Shim language is based on $\mathrm{C}$ (but is not a $\mathrm{C}$ subset) augmented with few constructs for concurrency, communication, and exceptions. Compared to the StreamDrive, Shim imposes many syntactic restrictions on the input language which makes porting existing reference applications more difficult. Furthermore, Shim does not address the runtime implementation issues, instead it relies on costly standard runtime support such as Pthreads library.

DOL implements the KPN execution model using cooperative protothreads [11]. While cooperative scheduling eliminates context-switching overhead and simplifies the runtime stack handling, the protothreads impose a number of important language restrictions leading to additional performance overhead and to artificial changes to the sequential reference code. Using the protothreads precludes implementation of real-time systems that may require preemptive scheduling. DOL assignment of actors to processors is static reducing load balancing ability. Unlike StreamDrive, DOL only implements the KPN execution model which leads to additional performance penalty compared to the dataflow execution because there are no firing rules: the sched- 
uler keeps trying to execute actors even when their blocking condition persists.

Like the work in [63] cited earlier, DOL takes advantage of shared memory in order to implement copy-free dataflow communication channels. In particular, DOL's windowed FIFOs [25] give actors direct access to data buffers to avoid copying. However, the windowed FIFO buffers cannot be shared between multiple actors, and do not support the broadcast and collect operations.

Gangwal et al [16] proposed the query/claim/release protocol, similar to the StreamDrive communication protocol. One important difference between the StreamDrive implementation and the query/claim/release protocol is that the StreamDrive synchronization counters count actual data bytes present in the communication buffers instead of tokens. This enables communicating actors to refer to different token sizes while still benefiting from an efficient synchronization support. Most importantly, the query/claim/release protocol do not allow buffers to be shared between multiple actors.

StreamDrive actors' ability to share data buffers is essential for reducing memory requirements and the communication overhead, but also for supporting the data parallelism. Zaki et al [64] developed Partial Expansion Graphs (PEGs) to help exploit the data parallelism in addition to functional/pipeline parallelism for SDF graphs. Similar to the StreamDrive, several instances of dataflow actors may be instantiated depending on relative load of the actor in terms of the execution time. Compared to StreamDrive, the PEG methodology has a number of important restrictions: (1) it is applicable to SDF graphs, (2) the data-parallel actors cannot have internal state, and (3) the dataparallel actor instances must execute in different processing cores. The work in [64] also develops a dynamic scheduling heuristics and shows that this scheduler performs better than the static one when actor execution loads are variable. However, their runtime implementation incurs higher performance overhead compared to StreamDrive: the synchronization and scheduling are ensured by a special buffer manager actor built on top of the underlying RTOS services, while StreamDrives' broadcast and collect synchronization is built into the scheduler itself. Finally, proposed Particle Swarm Optimization (PSO) approach for calculating the degree of the data parallelism for dataflow actors is general enough and can be applied on top of the StreamDrive as well.

Dynamic dataflow scheduling in the context of multicore systems has been studied by Michalska at al [34, 35]. The techniques proposed in these publications can be easily integrated with the StreamDrive distributed scheduler.
The above mentioned published research has not fully addressed the question of efficient execution of dynamic dataflow models, such as DDN and KPN, in small-scale clustered embedded architectures. Furthermore, they do not address the integration of specialized hardware blocks with programmable components in a single dataflow representation.

The integration of the application-specific hardware blocks has been previously addressed in the context of the high level synthesis (HLS) design flow. Several authors used RVC-CAL language as a single starting point for description of SW and HW components in a heterogeneous platform [47, 1, 2, 51]. Serot et Al [57] developed CAPH programming language for describing and implementing stream-processing applications on reconfigurable hardware, such as FPGAs. CAPH is based upon the dynamic dataflow model, supports an automated compilation producing VHDL code, and structurally reminds CAL.

These methodologies propose the software/hardware co-design flow that automates analysis, synthesis, optimization, and design space exploration for a given dataflow application. While targeting an applicationspecific solution, they pursue three design objectives: (1) higher degree of program analyzability and fast design cycle, (2) platform independent description that can be utilized for any implementation platform, and (3) rapid exploration of design alternatives. However, as explained earlier, CAL (and similarly, $\mathrm{CAPH}$ ) is a specialized language with limited code base. The mainstream programming languages for heterogeneous computing are $\mathrm{C}$ and $\mathrm{C}$-like languages. Porting a $\mathrm{C}$ application to CAL requires considerable effort and investment, comparable to developing a dynamic dataflow application from scratch.

On the other hand, we are interested in developing specialized hardware blocks for a particular target platform that can be reused accross a given application domain, eg. the convolution for image processing. This requires analyzing multiple applications and a "generic" hardware block development approach. The hardwaresoftware partitioning and architecture exploration in our work has been conducted using higher-level simulation, with hardware block models derived from reference $\mathrm{C}$ functions with minimal modifications of original code. The specialized hardware blocks in our work have been designed using traditional RTL development flow. If desired, in order to speed-up the design cycle and to make the design technology-independent, the HLS tools such as CatapultC can also be used for these hardware blocks implementation. 


\section{Target Platform}

StreamDrive targets small-scale clustered embedded architectures, where heterogeneous processing elements (PEs) are grouped into small clusters sharing a finite amount of resources including local memory. In this paper, we focus on programming a single cluster of such PEs. We are targeting image processing domain with real-time requirements of processing multiple image frames per second, we adopt the strategy of distributing computations of different image frames on different clusters. Thus, in a multicluster configuration, each cluster executes the same dataflow application applied to different image frame.

Fig. 1 shows the block diagram of our target architecture, the STMicroelectronics' ASMP cluster. It is composed by a number of programmable cores, specialized hardware blocks (HWPEs), and a DMA, all connected together to a shared Tightly-Coupled Data Memory (TCDM). The HWPEs are essential for achieving the required performance while keeping the cost and the power consumption low. In order to even further optimize power-efficiency of the system, the HWPEs can run each in their own different dedicated clock domain, thus allowing for the adjustment of their frequency in accordance with application requirements. Seamless integration of the hardware blocks is one of the important advantages of the StreamDrive framework.

The key element of the ASMP cluster is its logarithmic interconnect [45] that allows multiple concurrent accesses to the multi-bank TCDM memory. In order to minimize the number of stalls due to conflicting simultaneous accesses to the same bank, the banking factor (i.e. the ratio between the number of TCDM memory banks and the number of access ports), needs to be correctly dimensioned. Such shared memory organization, although it has a limited scalability, corresponds well to the small-scale cluster architecture that we target. Our experience, confirmed by other studies on similar architectures [8], shows that this type of interconnect can support up to 32 access ports, each with a throughput close to 32-bits/cycle with latency compatible with the RISC core internal pipeline, under the embedded IP target frequencies.

The connection between the HWPEs and the shared memory is ensured by the hardware block interface (I/F $0, . . \mathrm{I} / \mathrm{F} \mathrm{K}-1$ in the figure) that serves as a bridge for streaming hardware blocks. The programmable cores, the hardware block interface, and the DMA, all support the StreamDrive communication protocol based on shared memory - this creates a common infrastructure for the core-to-core, the core-to-hardware-block, or the hardware-block-to-hardware-block communication.

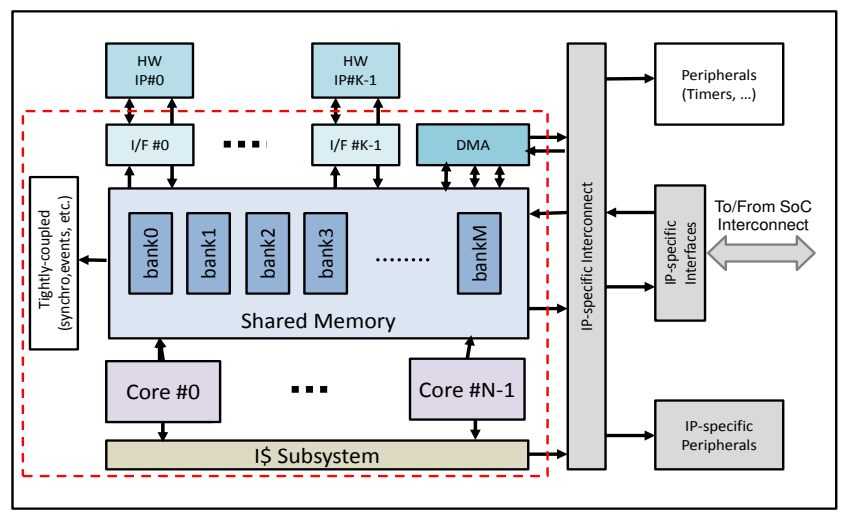

Fig. 1 The ASMP Cluster Block Diagram

The shared tightly-coupled memory organization is important for taking full advantage of the StreamDrive features.

The size of the TCDM has important impact on area-efficiency (GOPS/mm2) of the system: the larger the TCDM the lower area-efficiency. Generally, relatively small TCDM memory cannot fit the entire application working set. The StreamDrive cluster includes a DMA used for data movement between the TCDM and larger external off-chip memory. The DMA ensures additional function of synchronizing data transfers with the rest of the processing.

Finally, the ASMP cluster includes a small number of tightly-coupled peripherals aiming at accelerating synchronization, event handling, etc.

The current ASMP cluster implementation targets mobile image processing applications and includes 8 RISC processor cores running at relatively low frequency $(500 \mathrm{MHz})$, with $32 \mathrm{~K}$ of instruction cache each. The processor cores are extended with a small dedicated set of specialized instructions resulting in a $2-4 \mathrm{X}$ acceleration of important image processing functions ${ }^{1}$ The cluster also includes two hardware blocks: the Gaussian filter and the Scaler interpolation block, - along with $256 \mathrm{~KB}$ of the TCDM memory. Overall, one ASMP cluster delivers up to $8 \mathrm{Gops}$ at $500 \mathrm{MHz}$ not counting the hardware blocks, while the TCDM peak bandwidth reaches $32 \mathrm{~GB}$ per second. A predecessor of this architecture, featuring 4 clusters and 16 processors per cluster, with no hardware blocks, achieved power consumption of $2 \mathrm{~W}$ in $28 \mathrm{~nm}$ technology [33]. ASMP targets an even lower power and silicon area budget, position-

1 This set includes relatively generic instructions, such as a MAC4CLIP which performs SIMD multiplication on bytes of two input operands, saturates the two 16-bit results, and accumulates them with the result operand; as well as instructions dedicated to specific image processing functions, such as a XORSBCW, used in Support Vector Machine (SVM), which calculate the Hamming distance between two vectors. 
ing this platform well within the low-power, low-cost embedded profile.

Although the logarithmic interconnect technology constraints limit the scale of a tightly-coupled cluster to a couple of dozens of processing elements, multiple clusters can be put together allowing massive up-scaling in performance while maintaining the initial power- and area- efficiency. However, the multi-cluster aspects are out of this paper's scope.

As described in this section, our StreamDrive implementation leverages the tightly-coupled shared memory available in clustered platformes similar to ASMP. However, the StreamDrive framework can be retargeted to other architectures, for example, to distributed shared memory clusters. In this case, attention should be payed to efficiently implementing StreamDrive communication layer, in particular the broadcast and the collect connections (refer to next section). The StreamDrive scheduler is already distributed, however the global scheduling list will need to be implemented differently, perhaps using a work-stealing approach. As a bottom line, it is important to keep in mind that StreamDrive targets systems with small-scale clusters, not exceeding douzens of processing elements, as opposed to largescale massively parallel systems.

\section{StreamDrive Overview}

In this section, we discuss the StreamDrive API and the runtime system, and illustrate the incremental transformation flow of a sequential code into a dynamic dataflow implementation.

In the dataflow model of computation, an application is represented as a graph of actors ${ }^{2}$ connected by the communication channels, or dataflow buffers. The actors carry the actual computation while exchanging application-specific units of data, called tokens, over the communication channels. Tokens are written and read onto the communication channels in FIFO order. In StreamDrive, actors are connected to communication channels via input and output ports. Reading from an input port blocks the actor until all required tokens are available in the channel, and writing to an output port blocks the actor until enough empty room is available in the channel for the writing. For example, an actor performing an image filtering operation could be reading an input image line-by-line and writing the filtered image line-by-line. Then, the input and output communication tokens correspond to one line of the image.

2 In this paper, we also use term actor for the KPN processes for the sake of coherence.
Two major dataflow models of computation supported by the StreamDrive are the Kahn Process Networks and the Dynamic Dataflow Networks.

\subsection{The StreamDrive API}

The StreamDrive API is based on the C programming language and provides methods for defining the dataflow actors, for constructing the dataflow graph, and for controlling the runtime scheduler.

Each StreamDrive actor defines its private variables and its input and output ports. Actor ports specify the size of tokens exchanged on the given port. The actor's private variables and its communication ports are accessible from inside the actor functions via the THIS pointer. StreamDrive actors also define four basic functions: CONSTRUCTOR, DESTRUCTOR, INIT, and WORK. The CONSTRUCTOR and the DESTRUCTOR perform all actions required at actor creation and release time, in particular the actor ports are created inside the actor constructor function. The INIT function configures the actor for execution by initializing actor's internal state. Finally, the WORK implements the actor functionality.

The StreamDrive uses a copy-free communication protocol, leveraging on the shared TCDM available in hardware. The API defines four communication functions: reserve and push for writing the data to an output channel, and pop and release for reading the data from an input channel. Before writing into an output channel, a source actor must acquire a pointer to an available empty buffer entry via the reserve call. The function is blocking if no room is available inside the given output buffer. When the data have been written to the buffer, the source actor signals the availability of new tokens via the push call. On the destination side, an actor must acquire a pointer to an input token via the pop call before reading the data. The pop function is blocking if there is not enough available tokens in the FIFO. The destination actor does not need to make a copy of the data but instead can use data directly from the shared communication buffer. When the destination actor no longer needs the data, it must signal the source actor that the buffer can be reused via the release function.

The StreamDrive API also provides methods for creating actors and their ports, and for connecting actors via communication buffers. The StreamDrive graph description can be parameterized in number of actor instances and their connections. The API supports the configuration of dataflow graphs between executions by disabling actors, actor connections and by changing actor parameters. It is important to note that the application graph does not need to change depending on 
whether actors are implemented as software functions or as hardware blocks.

The StreamDrive model requires explicit management of the memory hierarchy, in particular of transferring data between the external and the local shared memory. Our experience is that streaming applications have regular memory access patterns and the advantages of explicit memory hierarchy management outweigh its inconveniences. The StreamDrive API implements a specific DMA support. A DMA function is similar to that of an actor: its implementation ensures that a synchronization is generated upon the DMA transfer completion in order to signal that a token is ready. In section 5 we show that using this mechanism, a very efficient hiding of external memory latency can be achieved.

Finally, the API provides a few functions for controlling the runtime scheduler. Of particular importance is the function for specifying the dataflow firing rules. A firing rule is specified by requiring certain number of free slots (output ports) or ready tokens (input ports) to be available in a given communication port before the next firing of the actor can take place. The firing rules can change for each new actor firing, supporting fully dynamic dataflow model. In the absence of firing rules, an actor behaves as a KPN process, possibly blocking during execution.

\subsection{The Incremental Parallelization Flow}

One important objective of the StreamDrive is to support the incremental transformation of a sequential reference code into an optimized dataflow form. In order to facilitate such transformation, the process is divided into a number of conceptually simple steps, each consecutive step is an incremental improvement over the previous one:

1. Identification of the dataflow part of the sequential application.

2. Identification of the dataflow actors and building the initial Kahn Process Network, KPN.

3. Refinement of the initial KPN by reducing actors granularity.

4. Identification and implementation of data parallel actors.

5. Conversion of the Kahn Process Network into the Dataflow Network by introducing the dataflow firing rules.

6. Optimization of the performance vs memory footprint trade-off.

The initial transformation of a sequential reference code into KPN form is facilitated by the fact that

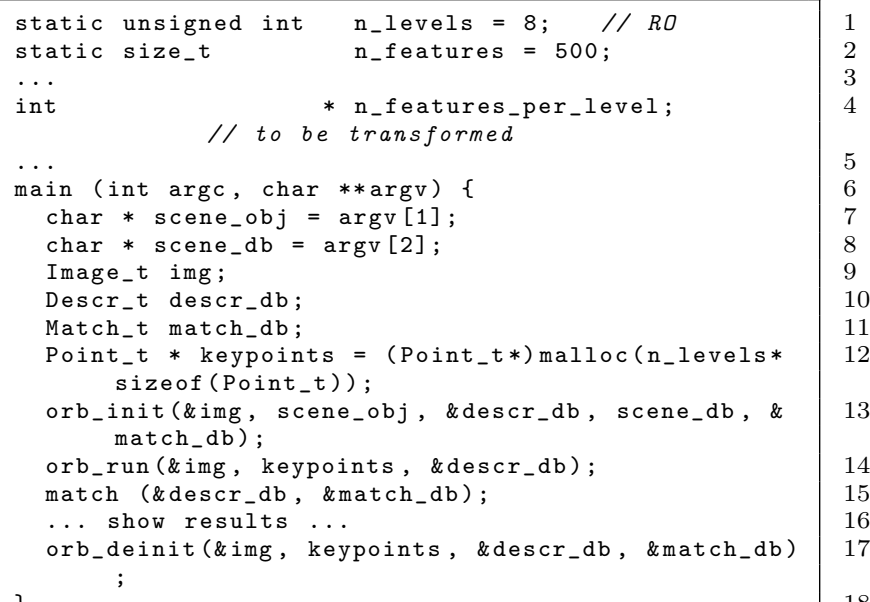

Fig. 2 Extract from the reference ORB application

streaming applications are typically structured into a sequence of processing kernels that roughly correspond to parallel Kahn processes. Transforming a sequential kernel into a Kahn process often requires minimal modifications to the code, consisting mostly of inserting KPN communication statements at appropriate places.

The biggest effort goes into achieving good performance vs memory footprint trade-off beyond the initial basic level. In this respect, the StreamDrive is not different from other parallelization approaches - usually a good understanding of the model is required in order to achieve high performance levels.

Importantly, all transformation steps can be performed incrementally, one actor at a time, allowing at each stage to debug and verify functional correctness of the transformation. In order to gain a more precise idea of the StreamDrive incremental parallelization flow, we illustrate the process using a real-life example. Figures 2 and 3 refer to the code for the Oriented 

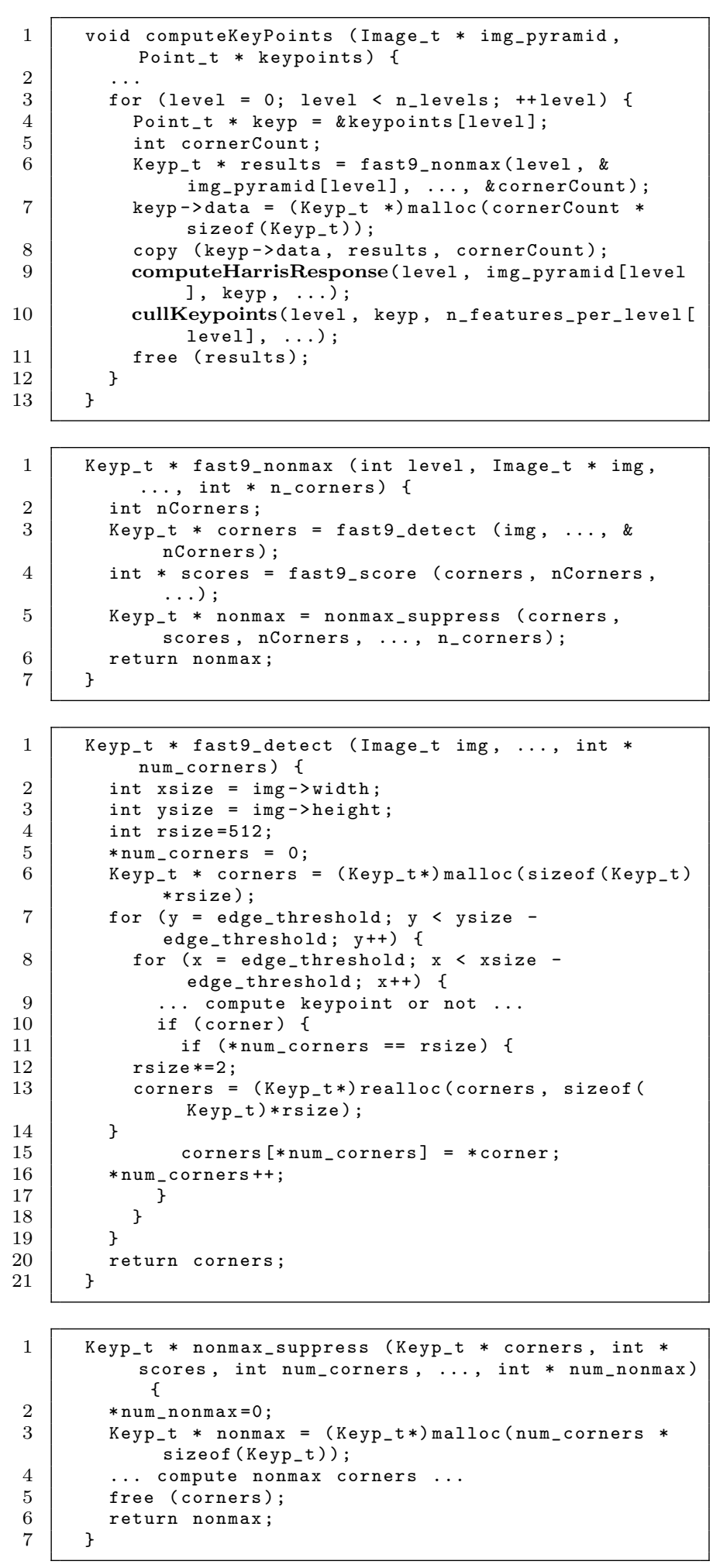

Fig. 3 Compute_Keypoints function from the ORB application

Fast and Rotated Brief (ORB) application. The ORB algorithm identifies a set of objects inside an image and matches their descriptors to the descriptors of ob-

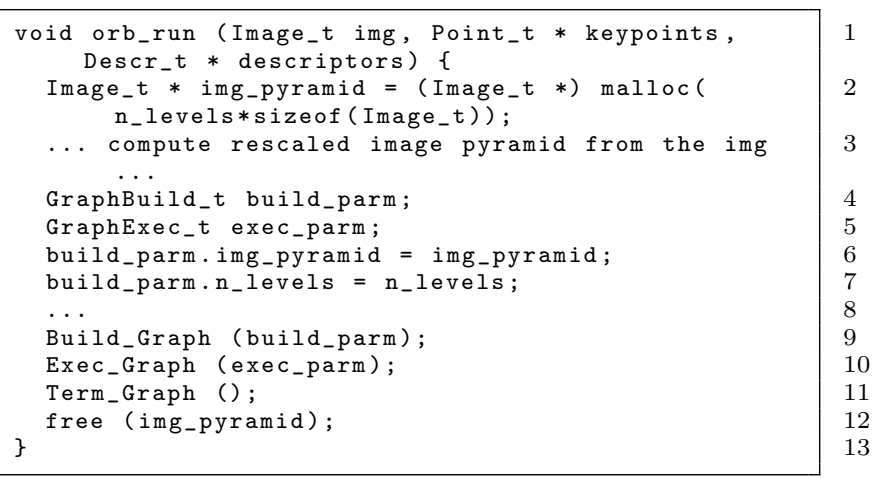

Fig. 4 The orb_run function modified to execute under the StreamDrive runtime.

jects in a trained database. The objects are identified by detecting keypoints of interest via the FAST algorithm [48]. The corner keypoints are selected via the nonmax suppression and then sorted using Harris response measure [24] to retain only the "best" keypoints. For these keypoints, the algorithm computes object orientation, and objects' BRIEF descriptor of the object associated with each keypoint. The descriptor computation requires the Gaussian filtered image. In order to be independent from the distance-to-object, processing is repeated over a series of images representing scaled down original image, the pyramid. ORB puts to evidence several important parallelization challenges: (1) ORB computation is irregular - some parts of the image may not have any keypoints, while others contain many; progressively reduced pyramid image sizes; (2) the nonmax and the sort computations are inherently serial; (3) the working set footprint is larger than can fit with the small L1 level memory, therefore our implementation extensively. uses DMA for transferring data to and from the external memory.

As a preparation step, the ORB application has been transformed from the floating-point version to the fixed-point suitable for an embedded implementation. The shown extract has been slightly amended for the purpose of the illustrating important points.

\subsubsection{Identification of the dataflow graph}

The ORB main function (line 6 in the top listing) receives the names of the image to process and of the objects database as arguments. Inside the main function, the orb_init loads the input image from a file; loads the trained objects database initializing the match_db for matching image objects vs the database objects; and initializes some global parameters. The orb_run computes the keypoints and the object descriptors. The match function compares the descr_db vs the match_db 
image pyramid in its input channel, and produces the array of cornerCount FAST keypoints with their FAST scores. The nonmax_suppress takes the keypoints generated by the fast9_detect as input and generates the set of corner keypoints by removing "uninteresting" keypoints from the set. The ComputeHarrisResponse reads these corners and computes the Harris response for each of them, which is a measure of "relevance" of each keypoint. The ComputeHarrisResponse output is the set of keypoints with their associated Harris response. The CullKeypoints performs the sorting of the keypoints with respect to their Harris response and reduces the keypoint set further by retaining at most the n_features_per_level best keypoints. From these remaining keypoints, the ComputeOrientation computes each keypoint orientation. The ComputeOrientation has two input channels, the keypoints generated by the CullKeypoints and the scaled input image from corresponding image pyramid level. The output of the ComputeOrientation is a set of keypoints with their associated orientation measure. The ComputeDescriptors takes two input channels as well, the output keypoints from the ComputeOrientation and the Gauss filtered input image. The ComputeDescriptors output is the final set of keypoints and their descriptors.

In order to introduce new actors, each processing kernel needs to be wrapped into the StreamDrive syntactic structure similar to the earlier ORB actor. Figure 6 shows as example the FAST actor corresponding to the fast9_detect kernel. Inside the actor .h file, the actor ports are declared, where the XXX_ESIZE defines port token size. The FAST input tokens are of type Image_t and output tokens are corners of type Keyp_t. Inside the .c file, the actor constructor and destructor functions create and destroy, respectively, the actor ports. The change to the original fast9_detect function is minimal and consists in inserting the StreamDrive communication primitives for writing the output data to the output port. At this point we do not use the input port yet, because we did not create an actor that can write the data to this port, therefore our FAST actor keeps reading the img-pyramid directly from external memory. The STREAM_OUT_RESERVE and the STREAM_OUT_PUSH implement the StreamDrive communication protocol. Thus, a reserve is called for every new corner and at the end all corners are pushed to the output channel. In order to communicate the number of corners to the downstream actor, the FAST reserves one header token at the beginning of the processing. When the number of corners is known at the end of the outermost loop, the header is pushed to the output channel

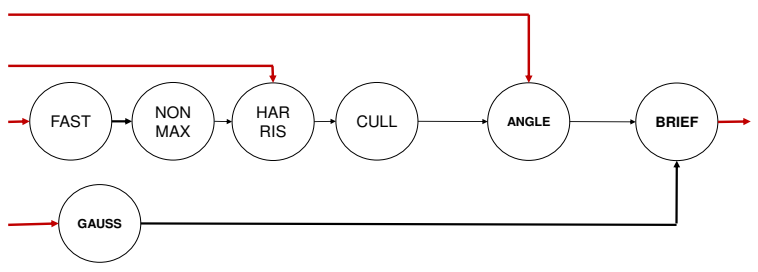

Fig. 7 Initial ORB dataflow graph: The actors correspond to the original kernels; FAST, GAUSS, HARRIS, and ANGLE read input image data directly from the external memory, BRIEF writes result descriptors directly to the external memory.

together with the corner tokens ${ }^{4}$. It is interesting to notice that using the streaming style communication allows us to get rid of inefficient malloc, realloc, and free calls.

The dataflow graph refinement is performed incrementally, one actor at a time, verifying the transformation correctness at each graph transformation. At the end of the process, the initial ORB actor is no longer needed and is removed from the graph. The resulting ORB graph is drawn in the Figure 7.

Figure 8 shows the corresponding Build_Graph function with seven actors. The STREAM_BIND function connects the output port of a source actor to the input port of the destination actor, while specifying the communication buffer depth and the memory level at which the buffer needs to be allocated. So far we have not addressed the memory size and actor granularity issues, therefore all buffers have been allocated in large external memory.

Notice that several ports remain unused in the current graph. These are ports that do not have actors to connect to. For example, the FAST actor input port which reads the input image from memory does not have a matching output port to connect to. Similarly, the GAUSS input port, the HARRIS and ANGLE ports that read input images from memory, as well as the BRIEF output port that writes final descriptors out to memory, do not have matching ports to connect to. All these ports correspond to input and output channels to the dataflow graph. This data initiate inside the external memory and need to be copied from this external memory to the L1 memory for processing.

We use the DMA engine to copy the external data to the L1 memory. For this, we use the StreamDrive DMA API. For the input channels, we introduce the new SRC actor which implements the DMA transfers. The SRC actor does not have input ports and has one output port to which data from the DMA transfer are sent. For transferring results from the BRIEF actor to the external memory, we add the StreamDrive DMA

4 any field of the Keyp_t structure can be used to communicate the number of corners. 


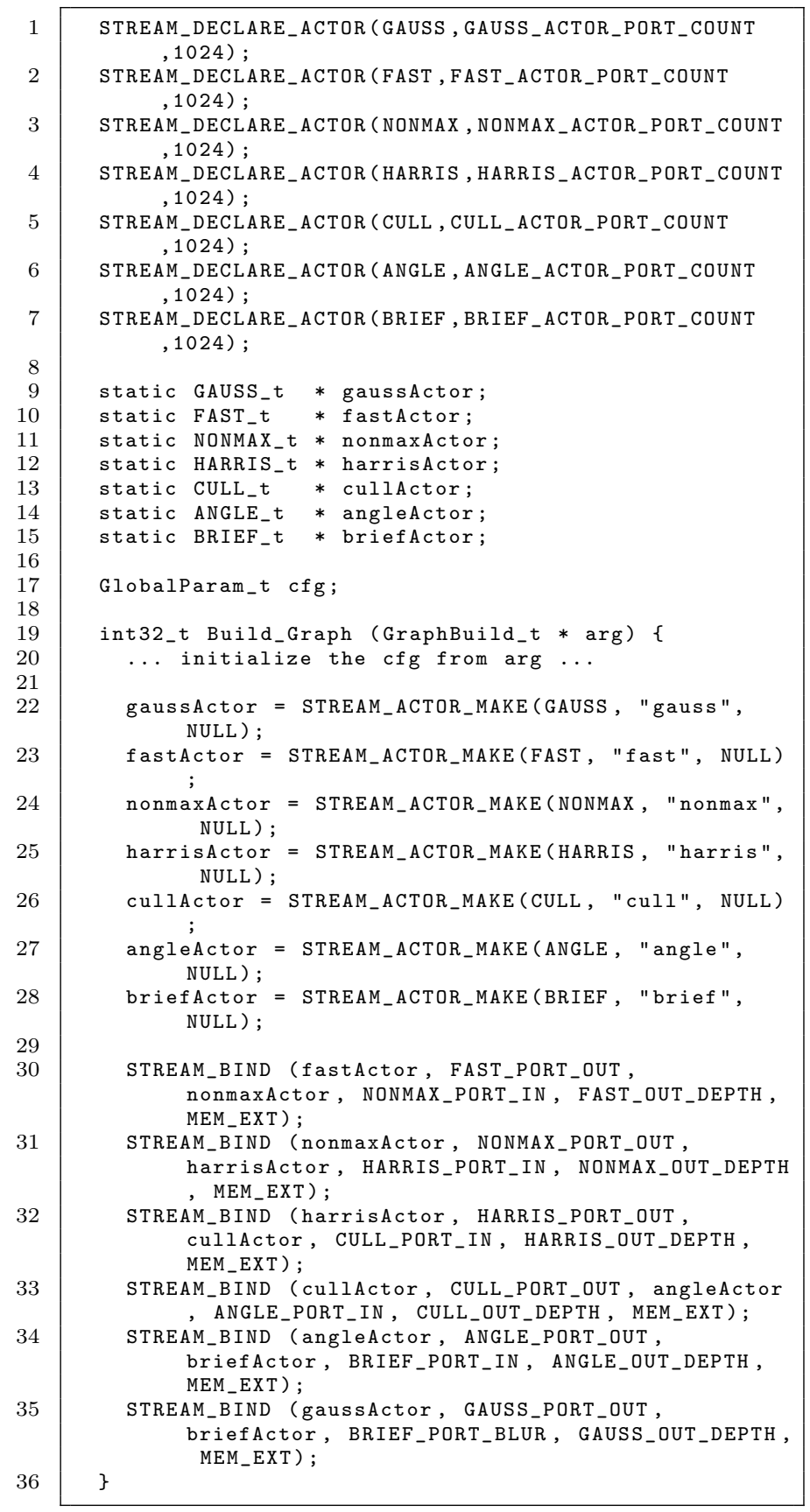

Fig. 8 Listing of the Build_Graph function that constructs the initial ORB graph.

API calls inside the BRIEF actor. Figure 9 shows the ORB graph with the SRC actor broadcasting the input image to several ORB actors.

At the end of this step, the initial dataflow graph is built with several dataflow actors identified. Following important points facilitate this transformation step: (1) the actor granularity of execution of the original application has been preserved; (2) we have avoided dealing with limited memory constraints by allocating all com-

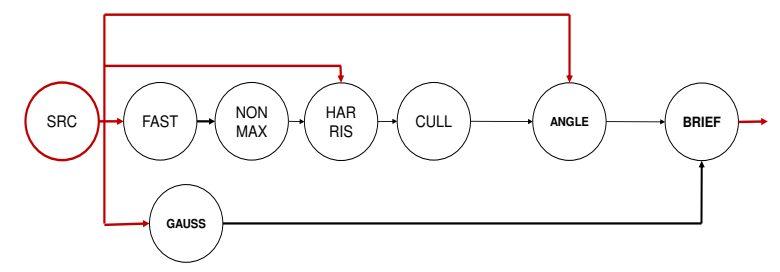

Fig. 9 ORB dataflow graph with the DMA actor added: the input image data are read via the DMA by the SRC actor and are broadcast to the FAST, GAUSS, HARRIS, and ANGLE actors. The BRIEF actor uses the StreamDrive DMA API for writing result descriptors to the external memory.

munication buffers in sufficiently large external memory; (3) the actor execution order corresponds to that of the original application because we have preserved the sequential code granularity and dependencies.

\subsubsection{The dataflow graph refinement}

The next transformation step is the dataflow graph refinement by reducing actor granularity so that dataflow communication buffers fit with limited L1 memory. The dataflow actor granularity refers to the amount of data that the actor needs for executing without being blocked, and is directly related to the size of actor input and output tokens.

\begin{tabular}{|c|c|c|}
\hline \# Actor & Port & Token size \\
\hline FAST & $\begin{array}{c}\text { IN } \\
\text { OUT }\end{array}$ & $\begin{array}{l}\text { One image line } \\
\text { One keypoint }\end{array}$ \\
\hline NONMAX & $\begin{array}{c}\text { IN } \\
\text { OUT }\end{array}$ & $\begin{array}{l}\text { One keypoint } \\
\text { One keypoint }\end{array}$ \\
\hline HARRIS & $\begin{array}{l}\text { IN } \\
\text { REF } \\
\text { OUT }\end{array}$ & $\begin{array}{l}\text { One keypoint } \\
\text { One image line } \\
\text { One keypoint }\end{array}$ \\
\hline CULL & $\begin{array}{c}\text { IN } \\
\text { OUT }\end{array}$ & $\begin{array}{l}\text { One keypoint } \\
\text { One keypoint }\end{array}$ \\
\hline ANGLE & $\begin{array}{l}\text { IN } \\
\text { REF } \\
\text { OUT }\end{array}$ & $\begin{array}{l}\text { One keypoint } \\
\text { One image patch } \\
\text { One keypoint }\end{array}$ \\
\hline GAUSS & $\begin{array}{c}\text { IN } \\
\text { OUT }\end{array}$ & $\begin{array}{l}\text { One image line } \\
\text { One image line }\end{array}$ \\
\hline BRIEF & $\begin{array}{l}\text { IN } \\
\text { BLUR } \\
\text { OUT }\end{array}$ & $\begin{array}{l}\text { One keypoint } \\
\text { One image patch } \\
\text { One descriptor }\end{array}$ \\
\hline
\end{tabular}

Table 2 Granularity of actors in the ORB application

Table 2 shows refined token sizes for the ORB graph actors. Notice that we have chosen to fetch the ANGLE and the BRIEF image data one patch at a time: a patch is a small area around each keypoint. Because patches for different keypoints may overlap, we end up fetching same image pixels multiple times. However, the alternative of keeping the keypoints in raster scan order and 


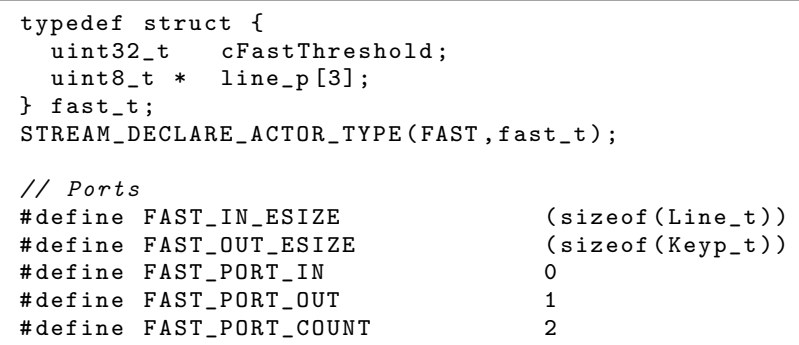

Fig. 10 The FAST actor KPN definition.

fetching reference image line by line led to poor performance.

Choosing actor granularity represents an important trade-off: finer granularity reduces the actor memory

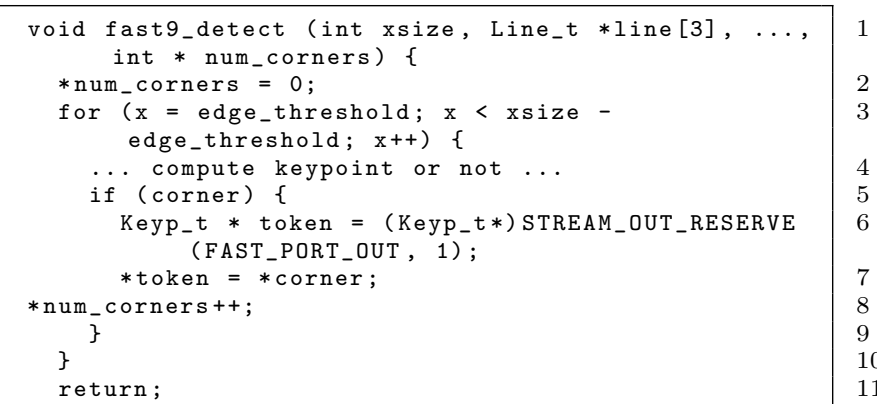

Fig. 11 The FAST actor fast9_detect function.

footprint while increasing the synchronization overhead ${ }^{5}$; coarser granularity suffers very little synchronization overhead but may require too much memory. Although granularity vs. performance is an application-specific trade-off, the parallelization should preserve application's natural granularity. In this context natural means as close to the intrinsic algorithm structure as possible. In an image processing application, choosing one image line as a dataflow token is natural because it corresponds to the second level in the image processing nested loop: (1) frame, (2) line, (3) pixel. As an alternative, sets of lines, tiles, or similar, are less natural in a sense that they are algorithm-specific, require some non-intuitive changes to the initial application code, and result is often radically different from the sequential algorithm.

Refining actors' granularity requires changing its WORK function. Figures 10 and 11 show the FAST actor with refined input granularity: the input token corresponds to one image line. Compared to the previous actor version from Figure 6 , the outermost level loop has been moved to the WORK function. Since the fast9_detect works on three lines at a time, we pass it a window of three lines, THIS->line_p, which is rotated on every iteration of the WORK function. The STREAM_IN_POP and the STREAM_IN_RELEASE implement the consumer side StreamDrive communication protocol.

Once the granularity of the actors has been reduced, the communication channels can be moved to the L1 memory. However, some channels may need to buffer too many tokens to fit with the L1 memory. For example, the SRC actor transfers the input image from the external to L1 memory one line at a time, while the FAST and the HARRIS actors consume these lines also one at a time. However, the ANGLE cannot start con-

5 The synchronization overhead includes actions required to verify the token availability, and the associated scheduler actions 


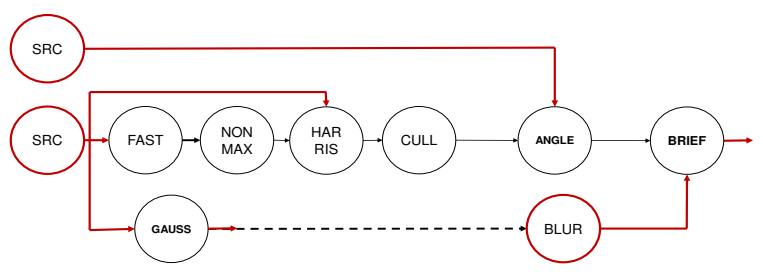

Fig. 12 Refined ORB dataflow graph: most communication buffers have been moved to the L1 memory. The SRC to HARRIS, and the GAUSS to BRIEF buffers do not fit with the L1 memory, therefore two additional DMA actors are added, the second SRC and the BLUR.

suming the input image lines until the entire image has been seen and processed by the CULL actor. Therefore, the communication channel needs to buffer the entire image and is, thus, too big to fit the L1 memory. In such cases, the communication channel buffer is allocated in the external memory with the DMA actors ensuring data transfer between the external and the L1 memories. The refined ORB dataflow graph is shown in Figure 12.

The refinement transformation step enables parallel execution for the first time: actors can execute their work-functions in parallel, synchronizing at reserve and at pop points.

\subsubsection{Adding application-specific hardware blocks}

Before further optimization and introduction of the firing rules, it is convenient to perform software-hardware partitioning at this point. For example, Table 3 shows the breakdown of ORB kernel's execution time from the original application (first image pyramid):

\begin{tabular}{|l|c|}
\hline Kernel & Execution Time, Mcycles \\
\hline fast9_detect & 3,77 \\
fast9_score & 0,39 \\
\hline nonmax_suppress & 0,32 \\
\hline ComputeHarrisResponse & 0,81 \\
\hline CullKeypoints & 0,34 \\
\hline ComputeOrientation & 0,55 \\
\hline ComputeGaussFiltering & 7,66 \\
\hline ComputeDescriptors & 2,09 \\
\hline Total & 15,93 \\
\hline
\end{tabular}

Table 3 The ORB execution time breakdown.

The Gaussian filtering kernel largely dominates the application execution time and, considering that filtering is a quite common function in image processing, is a good candidate for being implemented as an application-specific hardware block.

With StreamDrive, integration of application-specific hardware blocks does not require changing the dataflow graph. Instead, it is sufficient to change actor declaration from STREAM_DECLARE_ACTOR to the one declaring a hardware block, the STREAM_DECLARE_HWBLK. The StreamDrive runtime will transparently handle the hardware block actor during the execution.

\subsubsection{Data Parallelism}

The above transformation steps build a dataflow graph by identifying and exposing the functional parallelism, where multiple actors form execution pipeline over the input stream of data. Another important type of parallelism is the data parallelism. In data parallelism, multiple instances of the same actor are simultaneously created. The data parallelism leads to efficient execution when the computations are not data dependent and regular: (1) it is easy to identify and to expose, (2) it has lower parallelization overhead compared to the functional parallelism.

In the context of the StreamDrive, the data parallelism also allows to balance actors workload facilitating the work of the runtime scheduler. In the ORB application, the fast9_detect, the ComputeHarrisResponse, the ComputeOrientation, and the ComputeDescriptors kernels are regular and are easy to data parallelize. Parallelizing these kernels into a number of data-parallel instances has several advantages: (1) it balances the workloads of graph actors, and (2) it creates more actors for the scheduler to choose from. From the Table 3, the workload of the fast9_detect, is few times that of the nonmax_suppress or of the CullKeypoints kernels, and dividing its workload among several data parallel instances helps balancing the workload of all these actors.

Unlike the standard dataflow implementations, the StreamDrive includes the broadcast and the collect connections for efficiently supporting the data parallel actors (see section 4.4). The broadcast enables sharing of the input tokens by the data-parallel actors, while the collect allows sharing the output tokens. Using these connections, it is very easy to build data-parallel actors. Two data sharing strategies can be considered:

1. The single token data parallel actors work all on the same input or output token, but each on different part of it, for example a different part of an image line.

2. The multiple token data parallel actors work each on one of $\mathrm{N}$ tokens in parallel and synchronize on all $\mathrm{N}$ tokens simultaneously.

The data sharing strategies apply to individual input or output ports, and therefore it is perfectly possible to mix different data parallel strategies within the 


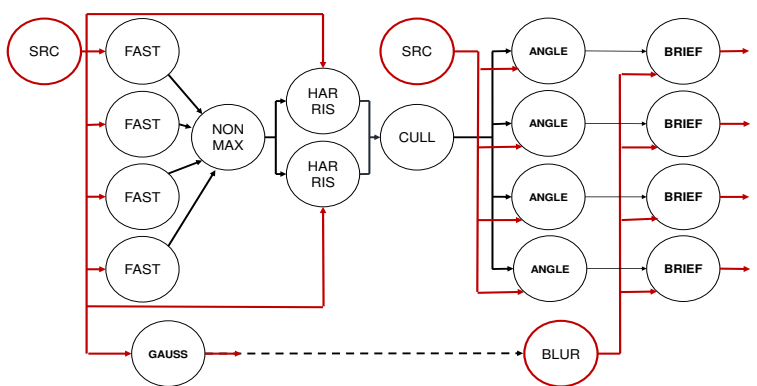

Fig. 13 The ORB dataflow graph with data-parallel actors: the FAST, the ANGLE and the BRIEF actors are replicated 4 times, and the HARRIS actor is replicated 2 times.

same actor, at the same time having channels which do not implement any data parallel sharing.

The Figure 13 shows the ORB dataflow graph with data-parallel actors. The broadcast connections are used to share input tokens of these actors. The collect connections are mostly optimized away, only the HARRIS actor data-parallel instances use the collect connection to share its output tokens. The FAST actor does not use the collect connection for its output because the downstream NONMAX actor needs the FAST corners to arrive in the raster scan order of the image. However, since the number of the corners in each image line is not known in advance, it is impossible for them to share the communication channel. As a solution, the NONMAX actor has 4 input ports, one for each upstream FAST actor, and reads them in a round-robin order ensuring that the FAST corners arrive in the raster scan order of the input image. Similarly, instead of using the collect connection for the output of the ANGLE actor, and then re-broadcast it to the BRIEF actors, we connect each ANGLE actor directly to the corresponding BRIEF actor, thus gaining efficiency.

The dataflow graph in Figure 13 shows the version with 4 FAST, 4 ANGLE, and 4 BRIEF data-parallel instances, as well as 2 HARRIS instances. Our implementation is parameterized in terms of the number of actors, their granularities, and the communication buffer sizes: it can be configured for $1 \mathrm{PE}$ with one instance of each actor up to 8 PEs with 8 instances of the FAST, ANGLE, and BRIEF actors.

Figure 14 shows the data parallel FAST actor. The new THIS->idx private field corresponds to the index of this data parallel instance among the 4 data parallel instances. This index is initialized via the actor constructor. The FAST actor implements the multiple token data parallel sharing in its input port. The changes to the actor's WORK function are minimal: the actors handle a shared rotating input window of 6 lines instead of 3 lines, while each actor processes only those lines that correspond to this actors' index. The actor

\begin{tabular}{|c|c|c|}
\hline \multicolumn{3}{|c|}{ typedef struct \{} \\
\hline \multicolumn{3}{|c|}{ uint8_t idx ; } \\
\hline \multicolumn{3}{|c|}{ uint 32 _t cFastThreshold; } \\
\hline \multicolumn{3}{|c|}{ uint8_t $*$ line_p $[6]$; } \\
\hline \multicolumn{3}{|c|}{ \} fast_t; } \\
\hline \multicolumn{3}{|c|}{ STREAM_DECLARE_ACTOR_TYPE (FAST, fast_t) ; } \\
\hline \multicolumn{3}{|l|}{ // Ports } \\
\hline \#define & FAST_IN_ESIZE & $\left(\operatorname{sizeof}\left(\right.\right.$ Line_t $\left.\left._{-}\right)\right)$ \\
\hline \#define & FAST_OUT_ESIZE & $(\operatorname{sizeof}($ Keyp_t $))$ \\
\hline$\#$ define & FAST_PORT_IN & 0 \\
\hline \#define & FAST_PORT_OUT & 1 \\
\hline \#define & FAST_PORT_COUNT & 2 \\
\hline
\end{tabular}

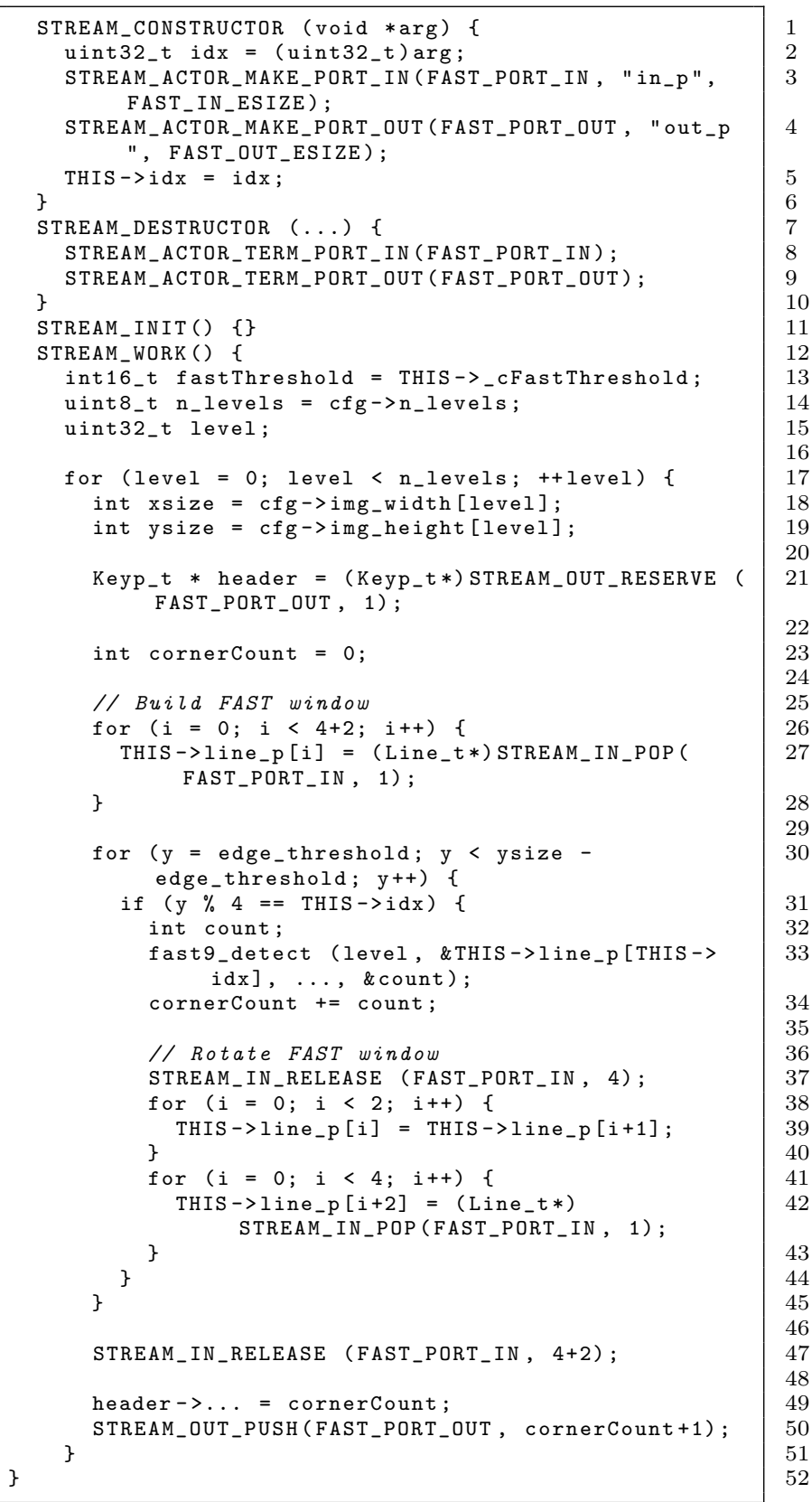

Fig. 14 Data-parallel version of the FAST actor

output is not changed since every FAST data parallel 


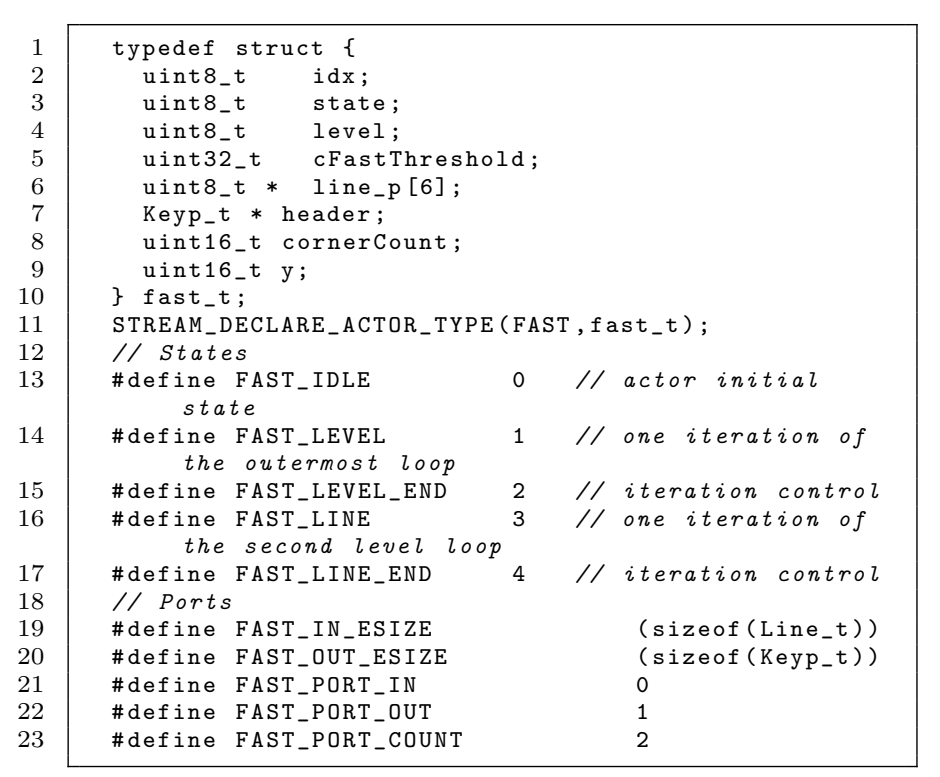

Fig. 15 ORB FAST dataflow actor definition.

instance handles its own (not shared) output channel. The fast9_detect function remains unchanged.

It is worth noticing that the StreamDrive offers great flexibility in connecting and synchronizing the data parallel actors. By buffering the input and output tokens, actors data parallel instances do not need to start and stop processing simultaneously, thus benefiting from the efficiency of pipelined execution. Finally, creating a few data parallel actors, we remain within the scope of a small-scale data parallelism (as opposed to the massive data parallelism with hundreds or thousands of parallel instances) matching well with the scale of the target architecture cluster.

\subsection{Optimizing Scheduling via Firing Rules}

Execution of the refined and parallelized dataflow graph can be optimized by introducing dataflow firing rules.

In KPN execution mode, software actors require the ability to suspend an actor on a blocked pop (or reserve), and to resume its execution when sufficient tokens (or empty FIFO entries) are available. Suspending and resuming actors implies costly context-switching. In the dataflow execution mode the firing rules give preconditions for actor execution by ensuring that there are enough input tokens (or room in output FIFOs) for the actor not to be blocked. Thus, dataflow mode allows the context-switch free, cooperative, scheduling.

In the dataflow mode, actor's WORK function is subdivided into a sequence of firings [9, 29]. During a firing, the actors reserve and pop tokens similar to the KPN mode, but the firing rules ensure that the actor is never

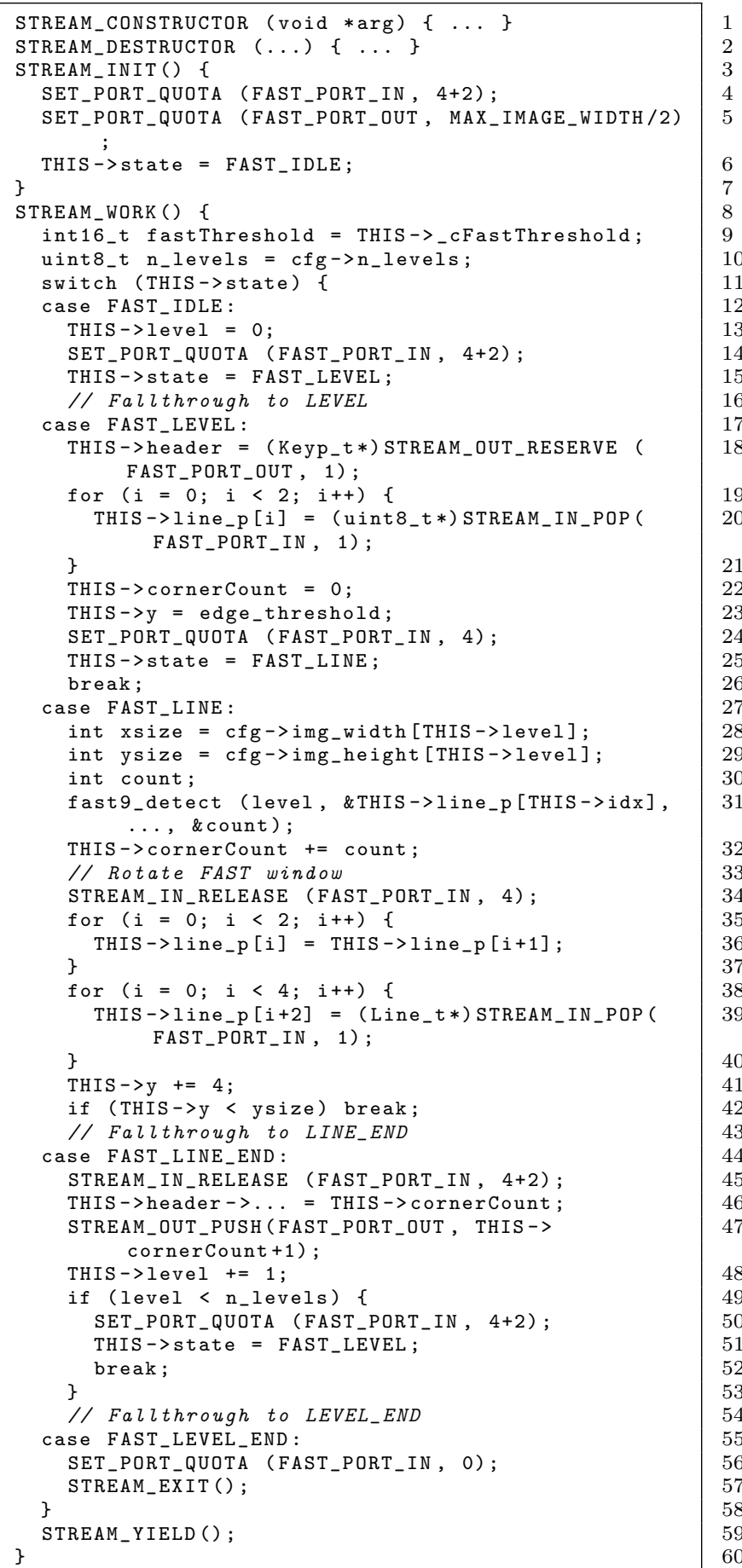

Fig. 16 ORB FAST actor with dataflow firing rules

blocked during the firing. When a firing is completed, actor returns control to the scheduler without requiring a context switch via the STREAM_YIELD call. The dataflow actor WORK function is "fired" by the scheduler until the STREAM_EXIT call signals the scheduler that actor completed its execution and does not require anymore firings. 
Introducing firing rules requires to once more change actor's WORK function. Figures 15 and 16 show the ORB FAST actor converted to the dataflow mode. The KPN version of the actor from the Figure 14 consisted of a loop nest with the outermost loop iterating over the image pyramid levels, the second level over the input image lines, and the innermost level iterating over the image pixels. First, we need to choose what one actor firing should be: the firing workload determines how many tokens this firing requires for the execution and therefore directly impacts actor's memory footprint. Thus, for our FAST actor we choose the second level loop, iterating over input image lines, as a firing unit. Next, all loops in the loop-nest above the chosen level need to be converted to a state machine. This conversion is relatively straightforward. The state machine states correspond to the loop-nest levels of the KPN actor: the FAST_IDLE corresponds to the initial state, the FAST_LEVEL and FAST_LEVEL_END to the outermost level loop, and the FAST_LINE and FAST_LINE_END to the second level loop. Before the WORK function yields the control to the scheduler, a transition to the next state needs to be specified by setting the private THIS-> state variable. In addition, the firing rules can be given for the next firing via the SET_PORT_QUOTA call. The SET_PORT_QUOTA function takes two arguments, the input or output port id and the number of tokens to expect in that port before the firing can take place. The initial state and the initial firing rules can be specified inside the actors' STREAM_INIT function. Notice that by default, unless set by the actor, the firing rules are not set and the actors' reserve and pop calls become blocking similar to the KPN execution mode.

One important point about converting the graph into the dataflow form is that all variables live across multiple actor firings need to be saved by the actor before the end of the firing and restored in the next firing. For this, such variables need to be added to actors' private state, similar to local variables level, header, y, and cornerCount from the FAST actor.

\subsubsection{Further refinement and optimization}

In the embedded domain, the cost of the system and the power consumption are directly related to the system memory size, and therefore reducing application memory footprint is very important. The dataflow program memory footprint depends on the communication buffers size and is finally related to the actors' granularity. The coarser the actor granularity, the bigger is the memory footprint. On the other hand, when the granularity of a program is very fine, the intrinsic overhead of the runtime has a high impact on efficiency. Thus, the optimization objective consists in finding the best trade-off between the communication buffer sizes and the parallelization overhead.

This step is the most time-consuming of the entire transformation process since the developer needs to choose from many different possibilities leading to different trade-off results. For example, we have noticed that processing the NONMAX, HARRIS, or CULL one keypoint per firing is inefficient because the amount of work per keypoint is small relative to the actor invocation overhead. One possibility that we explored was to combine the three actors together thus creating larger workload per keypoint. While this works well with smaller number of processing resources (less than 4 processing elements), when the number of processing resources increases, the resulting bulky actor is difficult to efficiently schedule and balance with other actors. On the other hand, we have noticed that several keypoint are usually simultaneously available for processing by the above actors. Therefore, we use the StreamDrive multitoken version of the communication API for increasing the firing working set of the actors and to reduce the parallelization overhead. Notice that the StreamDrive flow treats increasing the working set granularity as an optimization task within a well defined reference frame - number of tokens per actor firing. At the same time, preserving tokens natural granularity allows optimized application keep algorithmic description close to the original code.

As a general rule, the optimization process should first search for the possibility to combine actors together - this has additional benefits of reducing the overall buffer requirements since intermediate buffers between the combined actors can often be eliminated, and of reducing the schedulers' workload since fewer actors are active in the system. Then, the optimization should work to increase the number of tokens used in actors firings until an acceptable trade-off between the performance and the memory footprint is found.

This sections' example illustrates several important points from the StreamDrive:

- The StreamDrive incremental transformation flow facilitates parallelization of sequential applications into the dataflow implementation. For example, the original fast9_detect code incrementally undergoes relatively simple modifications during the transformation process: using the rotating window of image lines instead of the full image; addition of the StreamDrive communication primitives.

- The StreamDrive does not impose any specific language restrictions on reference code in order to be parallelized. 
- Unlike canonical dataflow, the StreamDrive allows usage of shared global variables. Shared variables are very efficient way of communicating in a shared memory environment and it facilitates porting existing sequential reference code. In our example, the FAST actor relies on global cfg for retrieving parameters such as image width and height, etc. These parameters are also used by other actors and, instead of duplicating the set of these parameters for each actor, they are implemented as a shared data structure. The coherent use of the shared data remains developer's responsibility.

- The StreamDrive runtime simultaneously supports two execution modes, the KPN and the dataflow execution. This is essential for enabling our incremental transformation flow.

Following sections describe important points of the StreamDrive communication layer and runtime system implementation.

\subsection{The StreamDrive Communication Layer}

In order to gain higher efficiency, StreamDrive relies on a fixed-buffer implementation, i.e. token sizes and buffer sizes need to be specified at graph construction time and cannot change during graph execution. The drawback of this is that deadlocks cannot be resolved at runtime. However, the experience is that most applications exhibit a regular communication behavior that allows software developer to quantify the capacity of the FIFO buffers such that deadlock will not occur. Nevertheless, the StreamDrive provides the runtime timeout service that allows detecting the deadlock condition. Upon detecting a deadlock, the StreamDrive gives debug information about the state of the dataflow graph which helps the developer to eliminate the deadlock.

A standard dataflow FIFO implementation where data must be copied from a source actor to the communication buffer and then from the communication buffer to the destination actor, causes a significant execution overhead. Instead, the StreamDrive implementation leverages the cluster shared memory and gives actors direct access to shared communication buffers avoiding memory copy operations. The direct access to communication buffers is enabled by using the StreamDrive communication protocol described in section 4.1.

The dataflow model of computation defines a single source and a single destination communication FIFO buffers. This is an essential requirement for ensuring the dataflow execution properties and correctness. On the other hand, this also creates a significant execution overhead: when a source actor is connected to multiple destination actors, a special copy actor needs to be inserted between them in order to copy-forward the data from the source to each destination. As a result, several copies of the same data must be made and several copy operations executed, one for each destination actor.

Instead, the StreamDrive API defines a special broadcast connection which allows one source actor and multiple destination actors to share a single FIFO buffer. A release operation on such buffer is valid when all destination actors have released the buffer.

Finally, the baseline dataflow model does not provide efficient support for data-parallelism. Typically, some sort of split and join actors need to be inserted around a data-parallel actor to copy-forward tokens in a round-robin order to multiple data-parallel actor instances. This leads to significant overhead: the memory overhead for holding multiple copies of the same token; the performance overhead for performing multiple copy operations and for scheduling the split and the join actors.

In StreamDrive, we avoid having these additional split and join actors by leveraging on the above broadcast connection and its symmetric collect connection. The collect allows multiple source actors to be connected to a single destination actor and share a communication buffer. A push operation on such buffer is valid when all source actors have signaled a ready token. A data-parallel actor, then, can be constructed by connecting multiple parallel actor instances via the broadcast connection to source actors and via the collect connections to destination actors. Sharing communication buffers gives a choice of data-parallel implementation: data-parallel actors may choose to process a sub-part of a single token each, or to process a different token each, whichever results in lower parallelization overhead.

The broadcast release and the collect push operations are internally supported by the StreamDrive runtime system. Therefore, there is no need to schedule these operations - the runtime knows when the broadcast release or the collect push needs to be executed. For example, a broadcast release will only be executed if the broadcasting output port is blocked on FIFO full condition. Such dedicated support to the broadcast and the collect connections ensures optimal runtime execution.

\subsection{The StreamDrive Runtime System}

The StreamDrive runtime system provides application with a communication layer and the dynamic scheduler. It is implemented as a user-level library avoiding costly system calls and enabling optimization such as inlining function calls, etc. 
The runtime system is fully distributed with regard to the processors - there is no one process dedicated to the runtime system duties. Instead, each processor concurrently (1) performs its own scheduling and (2) handles synchronization actions related to the actor being executed by the processor. As a result, the StreamDrive dataflow scheduler is fully dynamic: it assigns and schedules actors for execution dynamically at runtime. The scheduler uses a simple round-robin heuristic for selecting next actor to execute. The StreamDrive runtime system is still centralized from the point of view of the memory because the runtime system uses a single, global scheduling list. Our evaluation in section 5 shows that we achieved an efficient distributed implementation with respect to Amdahl's upper bound.

As explained earlier, StreamDrive supports two execution modes, the dynamic dataflow and the KPN. In the dynamic dataflow mode, actors run-to-completion and therefore one runtime stack per processing element can be used during the execution, and these runtime stacks are reasonably small and fit inside the shared TCDM memory. In the KPN mode, actor execution can be suspended on a blocking condition (they do not runto-completion) - therefore each actor requires its own dedicated runtime stack. Placing too many actor stacks inside the TCDM memory raises an important difficulty because this memory is relatively small. For example, the 8 processing elements version of ORB application (see section 5) has 30 actors. Given a stack size of $2 \mathrm{~K}$ per actor, the total stack memory requirement would be $60 \mathrm{~KB}$, which corresponds to almost a quarter of the total available TCDM memory in the ASMP cluster.

The individual actor stacks should be allocated in the larger external memory. On the other hand, placing the runtime stack inside the external memory with long access latency, leads to a very inefficient, low performance execution. We address this difficulty by implementing a stack spilling strategy. For this, we allocate one runtime stack per processing element inside the shared TCDM memory in both execution modes (the total number of these stacks is independent of the number of application actors). The individual actor stacks are also allocated in the larger external memory. During the execution, actors use the TCDM allocated runtime stack. When an actor gets blocked during the execution, a context switch occurs, where the actors' register context is saved to a location inside the external memory. Together with saving actors' register context, the current runtime stack is also spilled to the actors' external stack location. When a blocked actor resumes execution, its register context is restored, and also its stack content is reloaded to the runtime TCDM stack from the external stack location. Notice that in the dataflow execution mode, actors do not get blocked and no context switch and stack spilling are necessary.

Using the above stack-spilling strategy increases the cost of a context-switch: in addition to usual saving and restoring registers, the stack contents need to be saved and restored as well. In order to alleviate the problem, StreamDrive optimizes the KPN execution as follows: (1) our runtime scheduler minimizes the context-switch occurrences, (2) we have implemented optimized, handcrafted code for the context switch routines. Moreover, in our experiments we have observed that, typically, the total number of bytes of stack that need to be spilled is quite small, and penalty for stack spilling is comparable to that of register context switching (see discussion in section 5.5). As a result, this strategy remains more efficient compared to executing a program with runtime stack inside the external memory.

One side effect of the stack spilling approach is that actor assignment to processing elements in KPN mode is no longer dynamic. Indeed, because different processing elements' runtime stacks point at different addresses in the TCDM memory, a suspended actor can only resume its execution in the same processing element (address space) where it has been suspended. As a result, each KPN actor keeps execution in the same processing element where it has begun its execution. In order to optimize assignment of KPN actors to processing elements, StreamDrive provides a special API for assigning processor affinities to dataflow actors. However in general, we consider the KPN execution as an intermediate step during the incremental transformation process from a sequential reference code into a dataflow implementation with no context switching.

\section{Performance Evaluation}

To gain insight into the performance of the StreamDrive framework, we present the detailed analysis of the ORB application [49] described in detail in section 4. The first StreamDrive implementation is targeting small mobile camera systems with resolutions not exceeding VGA quality, 640x480 pixels. We present results obtained with a single, non-scaled, ORB pyramid frame containing 2,651 FAST keypoints and limiting the number of sorted keypoint to 764. This configuration is representative of the most demanding processing requirements for this scenario.

For our measurements we used a high level multithreaded simulator dedicated for modeling the ASMP platform (see section 3) with the objective to evaluate architectural trade-offs in earlier stage. Our simulation platform models the number and type of processing elements, how they communicate and how the 
memory is organized. The simulation platform is based on proprietory multithreaded design with a scheduling kernel which is responsible for the interaction with the architecture model to drive the simulation process. The platform integrates time-approximate simulators for programmable cores, which run as dedicated POSIX threads. The programmable cores are simulated with execution time approximation accuracy error below $10 \%$ compared to a cycle-accurate execution. All other components use a single POSIX thread and are scheduled in a cooperative fasion in order to speed-up the simulation. Finally, our simulation platform also models memory and interconnect conflicts giving a very reasonable simulation accuracy at a decent simulation speed.

The ORB actors' execution times are largely unbalanced, eg. the BRIEF requires double processing time compared to the ANGLE, the FAST is almost double processing time of the BRIEF, while NONMAX, HARRIS and SORT represent together less than $10 \%$ of the application processing time. Therefore, our parallelization strategy focused on balancing the actors execution times by creating multiple data-parallel actor instances, and pipelining actors in order to achieve the efficient execution as we described in section 4 .

Moving from a sequential reference $\mathrm{C}$ code to the initial dataflow graph is a smooth process. Starting with the initial dataflow graph, we have incrementally derived the optimized dataflow implementations for the application shown in the Figure 13. The biggest effort went into optimizing the dataflow graph with the objective to strike the optimal trade-off between the parallelization overhead and the memory footprint.

As explained earlier, our ORB graph implementation is parameterized in terms of the number of actors, actor granularities and the communication buffer sizes. In the parameterized dataflow graph, the number of data-parallel FAST, HARRIS, ANGLE, and BRIEF instances is configured depending on the number of processing cores available in the target platform. For example, the smallest ORB configuration targeting a single PE instantiates a single instance of each actor, while the biggest one targeting 8 PEs instantiates eight parallel instances of each, the FAST, ANGLE and BRIEF actors, and two parallel instances of the HARRIS actor. A choice of a particular dataflow graph configuration is dictated by the optimization objectives and should take into account the ease of developing, maintaining and evolving the parallelized code. For example, the dataflow graph in Fig. 13 resulted in best optimization trade-off for a cluster containing 4 processing elements and $64 \mathrm{~KB}$ of the TCDM memory, while actor implementation remains very close to the initial reference

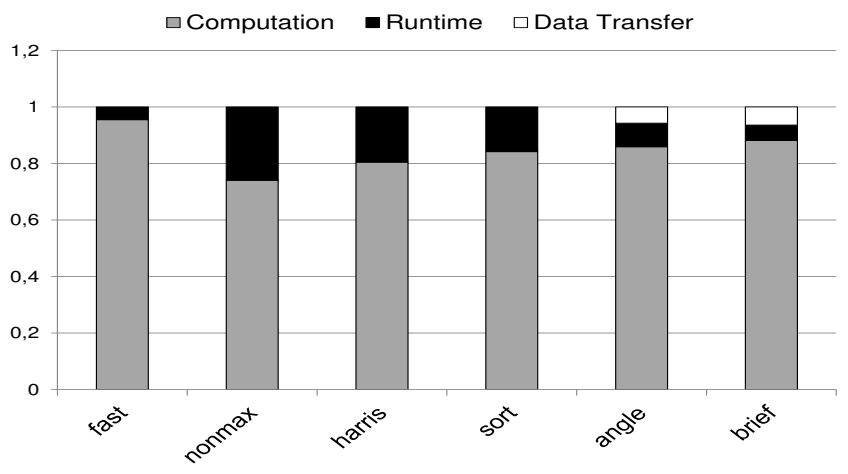

Fig. 17 ORB parallelization overhead: ratio of time spent in computation vs. data transfer and runtime tasks

$\mathrm{C}$ code. The capacities of communication buffers are a trade-off between the performance gain and the available TCDM memory size.

The order of application execution is deterministic, therefore debugging the dataflow code is similar to debugging the sequential one. One important difference concerns the dataflow deadlock that can occur if the communication buffers are incorrectly dimensioned. In our experience, dataflow applications exhibit a regular communication behavior so that quantifying the correct capacity of the buffers is relatively straightforward. A more formal approach for determining the dataflow buffer sizes has been proposed in [46], for example.

The following subsections analyze in details the StreamDrive parallelization overhead, memory footprint, and performance scaling under the optimistic assumption of external memory latency of 1 processor cycle and available external memory bandwidth of 8 bytes per processor cycle. We then show that StreamDrive maintains robust performance when we increase the external memory latency and reduce the available external memory bandwidth.

\subsection{Parallelization Overhead}

The parallelization overhead is a penalty paid for parallelizing an application. The StreamDrive parallelization overhead results from the runtime overhead including the RESERVE, PUSH, POP, and RELEASE functions, and from the DMA management for moving the data between external memory and the TCDM. This parallelization overhead does not include scheduling, which we evaluate later in this section. The parallelization overhead is scalable, i.e. from Amdahl's law perspective it contributes to the parallelizable part of the application.

In order to evaluate the StreamDrive parallelization overhead, we measure the performance of the ORB graph configured for $1 \mathrm{PE}$. The Figure 17 shows the 
breakdown of ORB actors execution into the computation, the runtime, and the data transfer management time.

The FAST performs heavy computation for each image pixel and therefore its parallelization overhead is small, $4.7 \%$ of actor's execution time. The NONMAX actor, on the other hand, has very little computation per pixel and suffers the heaviest parallelization overhead of all, 35.0\%. Similar to NONMAX, the HARRIS and the SORT actors perform relatively little computation per token and suffer from higher parallelization overheads, $24.2 \%$ and $18.7 \%$ respectively. One possibility that we explored is to merge the three actors into a single bigger actor. However, this only works well when parallelization degree is low (less than 4 processors) because the NONMAX and the SORT require sequential processing and the resulting actor is difficult to load balance with the rest of the application. We decided to favor better load balancing after having observed that the concerned actors' combined processing time represents less than $10 \%$ of the total application time (not counting the Gaussian filter). Finally, the ANGLE and the BRIEF actors include both the runtime and the data transfer management overhead, because they manage the DMA for transferring reference windows around each keypoint from external memory to the TCDM. Their runtime overhead is $9.2 \%$ and $5.9 \%$, while the data transfer management overhead is $6.2 \%$ and $6.7 \%$ respectively. Relatively high data transfer management overhead corresponds to many rather small transfer requests at the chosen dataflow actor granularity.

\subsection{Memory Footprint}

Application memory footprint determines how much memory the application needs for execution. The StreamDrive application memory footprint includes the application data, the run-time system footprint including the run-time stack, and the dataflow buffers.

In terms of the run-time system memory requirements, the debug version of the StreamDrive library uses 944 bytes of static data. It also needs 64 bytes of memory per actor in addition to actor private data, and up to 60 bytes per communication channel, depending on channel type. For comparison, an image line of a VGA image has a size of 640 bytes, while the smallest ORB keypoints buffer requires almost 300 bytes. Altogether, the ORB graph with 30 actors configured for $8 \mathrm{PEs}$ required in total less than $8 \mathrm{~KB}$ of memory for the run-time system. The stack contribution is application-specific and depends on the size of biggest stack that any one actor may require. As explained in section 4.5, the StreamDrive implementation allocates one runtime stack per processing element inside the TCDM. In ORB, we limit to $2 \mathrm{~KB}$ the stack space per actor, eg. the $8 \mathrm{PE} \mathrm{ORB}$ configuration required $16 \mathrm{~KB}$ of stack space for the 8 processing elements.

The application buffering requirements are determined by the actor granularity along with the size of the communication buffers. Every dataflow channel requires a minimal FIFO buffer size that ensures a deadlock free execution ${ }^{6}$. Additional buffer capacity beyond such minimal size helps improve performance by reducing scheduler overhead and by absorbing communication peaks when actor computation is irregular and unpredictable.

\begin{tabular}{|l|c|c|c|c|}
\hline \# PEs & $\min$ & $64 \mathrm{~KB}$ & $128 \mathrm{~KB}$ & $256 \mathrm{~KB}$ \\
\hline 1 & 37132 & 1.04 & 1.09 & 1.10 \\
\hline 2 & 45968 & 1.07 & 1.11 & 1.11 \\
\hline 4 & 63964 & 1.00 & 1.11 & 1.14 \\
\hline 8 & 99084 & & 1.00 & 1.18 \\
\hline
\end{tabular}

Table 4 Dataflow Performance Gain vs. Memory Footprint

Table 4 summarizes the ORB implementation memory footprint versus performance improvement associated with increasing the available memory size. For different ORB graph configurations from $1 \mathrm{PE}$ to $8 \mathrm{PEs}$, the min. column gives the minimal memory footprint, while other columns show the performance gain (ratio relative to the min.) that can be obtained by increasing the total memory size. From the table, it can be seen that performance gain due to adding more memory rapidly leads to diminishing returns. For example, with $4 \mathrm{PEs}$, the performance increase is $11 \%$ between $64 \mathrm{~KB}$ and $128 \mathrm{~KB}$, and only $3 \%$ when moving to $256 \mathrm{~KB}$. Note that the $8 \mathrm{PE}$ version of ORB does not fit in $64 \mathrm{~KB}$ memory with the minimal requirement close to a $100 \mathrm{~KB}$, due to large total number of actors leading to increased number of buffers.

\subsection{Performance Scaling}

The performance scaling of a parallel application indicates how much performance increases when more processing elements are added. In order to quantify the StreamDrive performance scaling, we measured the performance of the ORB graph configured for 8 PEs while varying the number of PEs. Figure 18 plots the resulting Amdahl's curve. We are observing the speedup very close to the theoretically optimal point. In the figure, the second line from the top corresponds to

6 Unless there is uncontrolled accumulation of tokens in a channel 


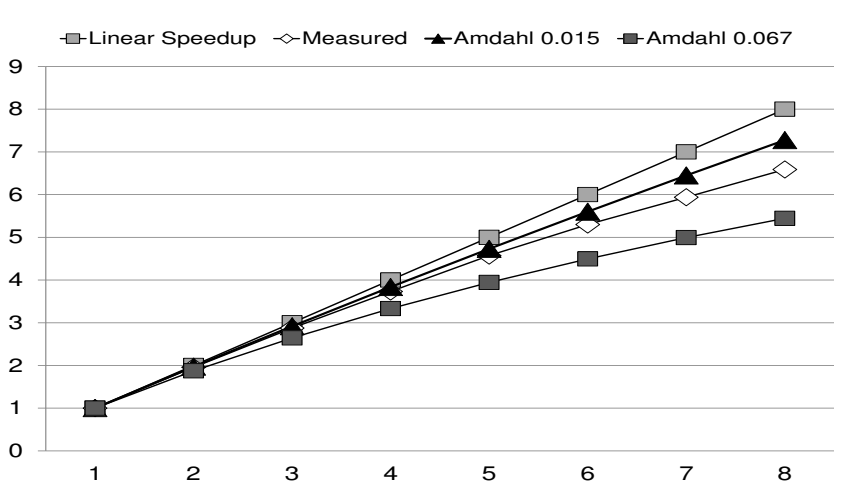

Fig. 18 ORB performance scaling: speed-up vs. number of PEs.

the Amdahl's speedup taking into account the $1.5 \%$ of non-parallelizable SORT part in the ORB application. The bottom line in the figure corresponds to the Amdahl's speedup taking into account additional 5.2\% of the scheduler overhead measured in a single PE, if it were non-parallelizable. The measured ORB speedup lies in between these two Amdahl's curves, showing that the StreamDrive scheduler is efficiently distributed over multiple PEs such that its non-parallelizable fraction is of the order of $1.5 \%$ of the total application execution time.

In order to quantify the efficiency of our broadcast and collect implementation, we have measured the time that application spends inside these functions. The broadcast and collect processing represent $4 \%$ and $3 \%$, respectively, of the StreamDrive scheduling overhead for scheduling 30 actors, i.e. the time for handling a broadcast or a collect is less than scheduling an actor, not even executing it.

Overall, we are observing the speedup very close to the theoretically optimal point. Note that this results have been achieved with a relatively small actor granularity. With larger input image sizes, the contribution of the SORT part would decrease as well as the scheduler overhead, resulting in speedups even closer to the linear.

It is interesting to compare our results with similar runtime environments. Compared to [22], the StreamDrive custom scheduler implementation is more efficient: among 30 ORB actors, we observe the average actor scheduling time of 161 cycles, versus 300 cycles for scheduling only 2 actors reported in [22]. The StreamDrive shared FIFO access is faster: less than 40 cycles versus 150. Finally, our ORB implementation with 30 actors required less than $8 \mathrm{~KB}$ of runtime system memory versus $9 \mathrm{~KB}$ for 2 small synthetic actors reported in [22].

Yviquel [63] reported performance scaling numbers of their dataflow implementation for MPEG-4 video

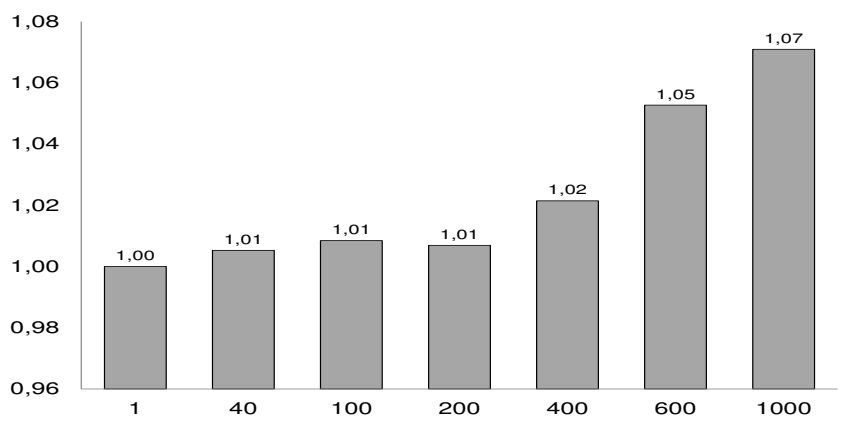

Fig. 19 ORB execution time increase vs. increased external memory latency

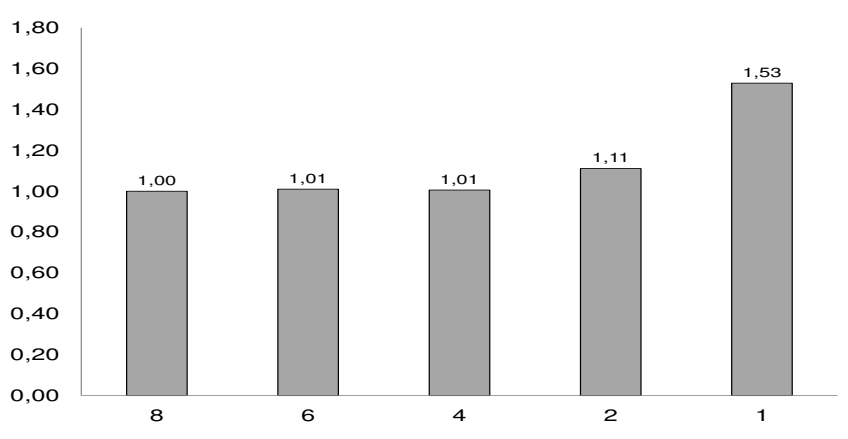

Fig. 20 ORB execution time increase vs. reduced external memory bandwidth

decoder. For comparison, with 10 processors their reported speedup is less than 6 times. The authors explain this relatively low speedup numbers by the limit of functional parallelism in the application. This confirms our experience showing the importance of the data parallelism.

The comparison with the work in [18] was not possible since the authors did not report their efficiency numbers.

\subsection{Performance w/r to External Memory}

The above evaluation of the StreamDrive implementation has been carried based upon an optimistic assumption of external memory latency of 1 processor cycle and available external memory bandwidth of 8 bytes per cycle. In a System-on-Chip (SoC) environment, where multiple IPs compete for the access to the DDR memory, this assumption is not valid. In order to evaluate the StreamDrive performance with varying external memory latency and bandwidth, we used the $8 \mathrm{PE}$ ORB configuration. This is the most demanding configuration in terms of external memory bandwidth because it requires data to be available simultaneously for a large number of actors. Notice that the relatively small ORB actor granularity results in many modest size DMA transfers, such as one image line of only 640 
bytes, or the ANGLE computation reference window of $31 \times 31$ bytes.

Figure 19 plots ORB performance change versus growing external memory latency from 1 to 1000 processor cycles. The figure shows that there is almost no performance degradation when external latency is smaller than 400 processor cycles. Furthermore, even when the latency is 1000 processor cycles, the performance degradation is only $7 \%$ versus the 1 cycle latency. Figure 20 plots the performance change when the available external memory bandwidth is reduced from 8 down to 1 byte per processor cycle. The performance starts to degrade visibly when the available bandwidth drops below 2 bytes per processor cycle. At $500 \mathrm{MHz}$, this corresponds to less than $2 \mathrm{~GB}$ per second, which is quite low for a typical SoC external memory.

Our evaluation results confirm that the StreamDrive performance holds well even under long external memory latency and limited available external memory bandwidth.

\subsection{KPN vs. Dataflow Trade-off}

Considering that the biggest parallelization effort is required by optimizing the dataflow graph after having introduced the firing rules, we have also compared the performance achievable with the KPN execution vs. the optimized dataflow execution.

In the KPN execution mode, the number of context switches during a program execution is proportional to the available buffer sizes: the bigger are dataflow FIFO buffers, the fewer are there context switches in the KPN mode. On the other hand, our dataflow scheduling heuristic is trying to fire a given actor as long as it remains enabled. Similarly to KPN, the number of times that the scheduler switches actors is also proportional to the dataflow buffer sizes. Therefore, we observe similar diminishing return behavior with the KPN execution: there is a point at which adding more buffer size to the dataflow graph leads to a negligible performance gain. Unlike the dataflow execution, the KPN performance results under the minimal buffer sizes are considerably worse than the performance at the diminishing return point. Table 5 illustrates this point:

\begin{tabular}{|l|c|c|c|c|}
\hline \# PEs & $\min$ & $64 \mathrm{~KB}$ & $128 \mathrm{~KB}$ & $256 \mathrm{~KB}$ \\
\hline 1 & 37132 & 1.45 & 1.48 & 1.50 \\
\hline 2 & 45968 & 1.24 & 1.42 & 1.44 \\
\hline 4 & 63964 & 1.02 & 1.32 & 1.36 \\
\hline 8 & 99084 & & 1.05 & 1.22 \\
\hline
\end{tabular}

Table 5 KPN Performance Gain vs. Memory Footprint

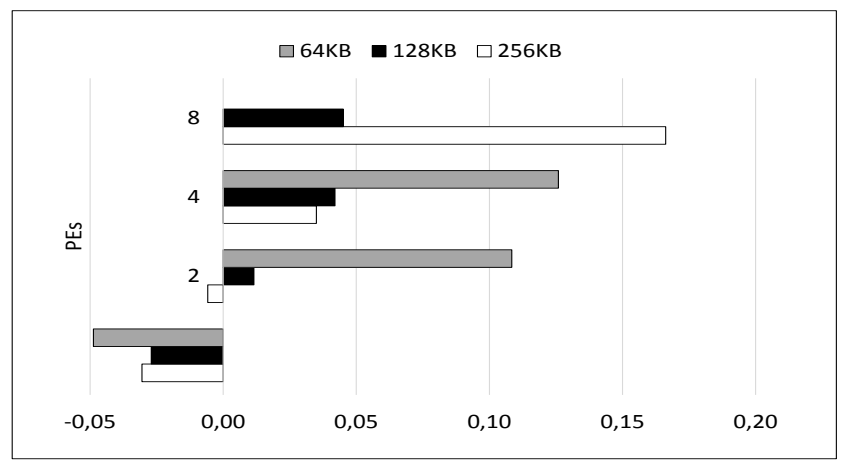

Fig. 21 ORB KPN vs. dataflow execution time, external memory latency 1 cycle

Unlike the DDN execution, the KPN execution is very sensitive to the external memory latency (and bandwidth). Figures 21 and 22 show the ratio of KPN vs. DDN execution cycles for ORB processing of one non-scaled VGA image when external memory latency is of 1 processor cycle (the external memory as as efficient as the TCDM) and 40 processor cycles, respectively. The Figures show measurements performed in different ASMP cluster configurations: TCDM memory size of 64,128 , and $256 \mathrm{~KB}$, and using 1, 2, 4, and 8 processing elements. While with external latency of 1 cycle (and few processing elements), the KPN performance may even be slightly better, the DDN clearly outperforms the KPN execution in all ASMP configurations when external memory latency is 40 processor cycles. The explanation is straightforward: the cost of the KPN context switch is directly related to the external memory access time because it is not possible to hide the context saving and restoring by performing it in parallel with other computation work. For example, there were 202 context switches during the KPN execution of the ORB in 1 processing element. With external latency of 1 processor cycle, they account for less than $2 \%$ of the total execution time. When the external memory latency increases to 40 cycles, these context switches account for $10 \%$ of the total execution time. Because the KPN performance is much more affected by the external memory latency than the performance of the DDN execution, the DDN would be a better choice for real embedded systems, where the external memory latency is often a bottleneck.

The KPN performance scales worse than the DDN performance when the number of processing elements increases. In Figure 21, the KPN performance is even slightly better than the DDN, less than $5 \%$, with 1 processing element. When 8 processing elements are used, the DDN outperforms the KPN by up to $18 \%$. The explanation is twofold: (1) the dynamic assignment of dataflow actors to processing elements outperforms the 


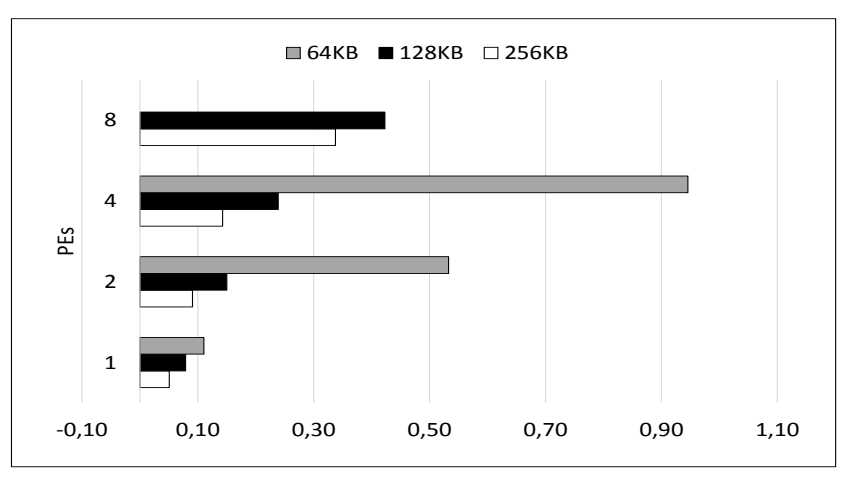

Fig. 22 ORB KPN vs. dataflow execution time, external memory latency 40 cycles

fixed KPN assignment, and (2) the relative contribution of the KPN scheduler is increasing faster than the contribution of the dataflow scheduler with more parallel execution.

To put the above performance measurements in prospective, notice that the execution time for processing one non-scaled VGA frame should not exceed $1,6 \mathrm{M}$ cycles at $500 \mathrm{MHz}$ operating frequency in order to achieve the real-time performance of 30 frames per second ${ }^{7}$. This level of performance can only be achieved with 8 PEs and under the dataflow execution mode. Thus, the effort spent in optimizing the dataflow graph is certainly necessary in order to achieve the target realtime objective.

\section{Conclusion and Future Work}

The StreamDrive framework implements the dynamic dataflow computing model. Two main contributions of the StreamDrive framework are: (1) simultaneous support for the KPN and the Dataflow execution modes, which enables the incremental parallelization flow starting with sequential reference code, and (2) an efficient runtime implementation in a resource-constrained embedded computing platform. StreamDrives' distributed runtime system provides low overhead, good scalability, and is robust versus limiting external memory bandwidth. The experience with the ORB application shows that StreamDrive is an efficient approach for parallelizing and executing embedded streaming applications.

The StreamDrive is a work in progress. The aspects of StreamDrive that need to be further investigated are primarily related to automating the optimization of the dataflow graph, and improvements to the runtime scheduler.

7 This real-time requirement also takes the match part of the application into account
Acknowledgements This research was partially funded by the H2020 Project Opecomp (CA 732631) and by the ERCADG Project Multitherman (CA 291125). Authors would also like to thank the ST Microelectronics' Embedded Computing Systems management for supporting this research.

\section{References}

1. Bezati E (2015) High-level synthesis of dataflow programs for heterogeneous platforms: design flow tools and design space exploration. PhD thesis, COLE POLYTECHNIQUE FDRALE DE LAUSANNE

2. Bezati E, Brunet SC, Mattavelli M, Janneck JW (2016) High-level system synthesis and optimization of dataflow programs for mpsocs. In: Matthews MB (ed) ACSSC, IEEE, pp 417-421

3. Bhattacharya B, Battacharyya S (2001) Parameterized dataflow modelling for dsp systems. IEEE Transactions on Signal Processing 49(10):2408 2421

4. Bhattacharyya SS, Deprettere EF, Leupers R, Takala J (eds) (2013) Handbook of Signal Processing Systems. Springer

5. Bilsen G, Engels M, Lauwereins R, Peperstraete JA (1995) Cyclo-static data flow. In: ICASSP, vol 5, pp $3255-3258$

6. Buck JT (1994) A dynamic dataflow model suitable for efficient mixed hardware and software implementations of dsp applications. In: HSCD Workshop, pp 165-172

7. Cockx J, Denolf K, Vanhoof B, Stahl R (2007) Sprint: A tool to generate concurrent transactionlevel models from sequential code. EURASIP Journal on Applied Signal Processing 1:213

8. Dehyadegari M, Marongiu A, Kakoee M, Benini L, Mohammadi S, Yazdani N (2012) A tightly-coupled multi-core cluster with shared memory hw accelerators. In: ISCAMOS, pp 96-103

9. Dennis J (1974) First version data flow procedure language. Tech. Rep. MAC TM61, MIT Laboratory for Computer Science

10. de Dinechin BD, Ayrignac R, Beaucamps PE, Couvert P, Ganne B, de Massas PG, Jacquet F, Jones S, Chaisemartin NM, Riss F, Strudel T (2013) A clustered manycore processor architecture for embedded and accelerated applications. In: HPEC, IEEE, pp 1-6

11. Dunkels A, Schmidt O, Voigt T, Ali M (2006) Protothreads: simplifying event-driven programming of memory-constrained embedded systems. In: Sensys, pp 29-42 
12. Edwards SA, Tardieu O (2006) Shim: A deterministic model for heterogeneous embedded systems. IEEE Transactions on Very Large Scale Integration (VLSI) Systems 14(8):854-867

13. Edwards SA, Vasudevan N, Tardieu O (2008) Programming shared memory multiprocessors with deterministic message-passing concurrency: Compiling shim to pthreads. In: Sciuto D (ed) DATE, ACM, pp 1498-1503

14. Eker J, Janneck J (2002) Caltrop-language report (draft). Technical memorandum, Electronics Research Lab, Department of Electrical Engineering and Computer Sciences, University of California at Berkeley California, Berkeley, CA 94720, USA, http://www.gigascale.org/caltrop

15. Eker J, Janneck JW (2012) Dataflow programming in cal - balancing expressiveness, analyzability, and implementability. In: Asilomar Conference on Signals, Systems and Computers, pp 1120-1124

16. Gangwal OP, Nieuwland A, Lippens PER (2001) A scalable and flexible data synchronization scheme for embedded hw-sw shared-memory systems. In: Hermida R, Aboulhamid EM (eds) ISSS, ACM / IEEE Computer Society, pp 1-6

17. Gautier T, Besseron X, Pigeon L (2007) Kaapi: A thread scheduling runtime system for data flow computations on cluster of multi-processors. In: PASCO, pp 15-23

18. Gebrewahid E, Yang M, Cedersjö G, Abdin ZU, Gaspes V, Janneck JW, Svensson B (2014) Realizing efficient execution of dataflow actors on manycores. In: EUC, pp 321-328

19. Geilen M, Basten T (2003) Requirements on the execution of kahn process networks. In: Degano $\mathrm{P}$ (ed) ESOP, Springer, Lecture Notes in Computer Science, vol 2618, pp 319-334

20. Girault A, Lee B, Lee EA (1999) Hierarchical finite state machines with multiple concurrency models. Computer-Aided Design of Integrated Circuits and Systems, IEEE Transactions on 18(6):742-760

21. Goubier T, Sirdey R, Louise S, David V (2011) $\Sigma$ C: A programming model and language for embedded manycores. In: ICA3PP, pp 385-394

22. Haid W (2010) Design and performance analysis of multiprocessor streaming applications. $\mathrm{PhD}$ thesis, ETH, Zurich

23. Haid W, Schor L, Huang K, Bacivarov I, Thiele L (2009) Efficient execution of kahn process networks on multi-processor systems using protothreads and windowed fifos. In: ESTIMedia, pp 35-44

24. Harris C, Stephens M (1988) A combined corner and edge detector. In: Proceedings of the 4th Alvey Vision Conference, pp 147-151
25. Huang K, Grunert D, Thiele L (2007) Windowed fifos for fpga-based multiprocessor systems. In: ASAP, pp 36-41

26. JT B (1993) Scheduling dynamic dataflow graphs with bounded memory using the token flow model. $\mathrm{PhD}$ thesis, Department of Electrical Enginnering and Computer Science, University of California at Berkeley

27. Kahn G (1974) The semantics of a simple language for parallel programming. In: IFIP Congress

28. de Kock EA, Smits W, van der Wolf P, Brunel JY, Kruijtzer W, Lieverse P, Vissers KA, Essink G (2000) Yapi: Application modeling for signal processing systems. In: DAC, pp 402-405

29. Lee E (1997) A denotational semantics for dataflow with firing. Memorandum UCB/ERL M97/3, Electronics Research Laboratory, U. C. Berkeley

30. Lee EA, Messerschmitt DG (1987) Synchronous data flow. Proceedings of the IEEE 75(9):12351245

31. Mattavelli M, Amer I, Raulet M (2010) The reconfigurable video coding standard [standards in a nutshell]. IEEE Signal Processing Magazine 27(3):159 167

32. Mattavelli M, Raulet M, Janneck JW (2013) Mpeg reconfigurable video coding. In: Bhattacharyya SS, Deprettere EF, Leupers R, Takala J (eds) Handbook of Signal Processing Systems, Springer, pp 281-314

33. Melpignano D, Benini L, Flamand E, Jego B, Lepley T, Haugou G, Clermidy F, Dutoit D (2012) Platform 2012, a many-core computing accelerator for embedded socs: performance evaluation of visual analytics applications. In: DAC, pp 1137-1142

34. Michalska M, Bezati E, Brunet SC, Mattavelli M (2016) A partition scheduler model for dynamic dataflow programs. In: Connolly M (ed) ICCS, Elsevier, Procedia Computer Science, vol 80, pp $2287-2291$

35. Michalska M, Zufferey N, Boutellier J, Bezati E, Mattavelli M (2016) Efficient scheduling policies for dynamic data flow programs executed on multicore. In: 11th International Meeting on Logistics Research

36. NVIDIA (2010) Next generation cuda compute architecture: Fermi - white paper. http://www.nvidia.com

37. Olofsson A, Nordström T, Ul-Abdin Z (2014) Kickstarting high-performance energy-efficient manycore architectures with epiphany. In: Asilomar Conference on Signals, Systems and Computers, IEEE, pp 1719-1726 
38. Orozco D, Garcia E, Pavel R, Khan R, Gao G (2011) Tideflow: The time iterated dependency flow execution model. In: Workshop on Data-Flow Execution Models for Extreme Scale Computing (DFM), pp 1-9

39. Pelcat M, Desnos K, Heulot J, Guy C, Nezan JF, Aridhi S (2014) Preesm: A dataflow-based rapid prototyping framework for simplifying multicore dsp programming. In: EDERC, pp 36-40

40. Pimentel AD (2008) The artemis workbench for system-level performance evaluation of embedded systems. International Journal of Embedded Systems 3(3):181-196

41. Plishker W, Sane N, Kiemb M, Anand K, Bhattacharyya SS (2008) Functional dif for rapid prototyping. In: IEEE International Workshop on Rapid System Prototyping, IEEE Computer Society, pp $17-23$

42. Plishker W, Sane N, Bhattacharyya SS (2009) A generalized scheduling approach for dynamic dataflow applications. In: Benini L, Micheli GD, Al-Hashimi BM, Mller W (eds) DATE, IEEE, pp 111-116

43. Plurality (2011) Plurality hypercore. http://www.plurality.com

44. Pop A, Cohen A (2013) Openstream: Expressiveness and data-flow compilation of openmp streaming programs. ACM Transactions on Architecture and Code Optimization 9(4):53

45. Rahimi A, Loi I, Kakoee MR, Benini L (2011) A fully-synthesizable single-cycle interconnection network for shared-11 processor clusters. In: Design, Automation \& Test in Europe Conference \& Exhibition (DATE), 2011, IEEE, pp 1-6

46. Rahman AAHA, Brunet SC, Alberti C, Mattavelli M (2014) A methodology for optimizing buffer sizes of dynamic dataflow fpgas implementations. In: ICASSP, IEEE, pp 5003-5007

47. Rahman AAHBA (2014) Optimizing dataflow programs for hardware synthesis. PhD thesis, COLE POLYTECHNIQUE FDRALE DE LAUSANNE

48. Rosten E, Porter R, Drummond T (2010) Faster and better: A machine learning approach to corner detection. Pattern Analysis and Machine Intelligence, IEEE Transactions on 32(1):105-119

49. Rublee E, Rabaud V, Konolige K, Bradski G (2011) Orb: An efficient alternative to sift or surf. In: ICCV, pp 2564-2571

50. Sane N, Hsu CJ, Pino JL, Bhattacharyya SS (2010) Simulating dynamic communication systems using the core functional dataflow model. In: ICASSP, IEEE, pp 1538-1541
51. Sau C, Meloni P, Raffo L, Palumbo F, Bezati E, Brunet SC, Mattavelli M (2016) Automated design flow for multi-functional dataflow-based platforms. Signal Processing Systems 85(1):143-165

52. Schwambach V, Cleyet-Merle S, Issard A, Mancini S (2015) Estimating the potential speedup of computer vision applications on embedded multiprocessors. CoRR abs/1502.07446

53. Shen C, Plishker W, Bhattacharyya SS (2012) Dataflow-based design and implementation of image processing applications. Multimedia Image and Video Processing pp 609-629

54. Sriram S, Bhattacharyya SS (2009) Embedded multiprocessors: Scheduling and synchronization. CRC press

55. Stoutchinin A, Benini L (2017) Stream drive: A dynamic dataflow framework for clustered embedded architectures. In: Conf. Computing Frontiers, ACM, pp 1-8

56. Stuijk S, Geilen M, Thelen B, Basten T (2011) Scenario-aware dataflow: Modeling, analysis and implementation of dynamic applications. In: International Conference on Embedded Computer Systems, pp 404-411

57. Srot J, Berry F, Bourrasset C (2016) High-level dataflow programming for real-time image processing on smart cameras. Journal of Real-Time Image Processing 12(4):635-647

58. Ul-Abdin Z, Yang M (2015) A radar signal processing case study for dataflow programming of manycores. Journal of Signal Processing Systems pp 1-14

59. Vasudevan N, Edwards SA (2009) Celling shim: Compiling deterministic concurrency to a heterogeneous multicore. In: ACM Symposium on Applied Computing, pp 1626-1631

60. Vrba Z, Halvorsen P, Griwodz C, Beskow P, Espeland H, Johansen D (2013) The nornir run-time system for parallel programs using kahn process networks on multi-core machines - a flexible alternative to mapreduce. The Journal of Supercomputing 63(1):191-217

61. YarKhan A (2012) Dynamic task execution on shared and distributed memory architectures. PhD thesis, the University of Tennessee, Knoxville

62. Yviquel H, Sanchez A, Jskelinen P, Takala J, Raulet M, Casseau E (2014) Efficient software synthesis of dynamic dataflow programs. In: ICASSP, IEEE, pp 4988-4992

63. Yviquel H, Sanchez A, Jskelinen P, Takala J, Raulet M, Casseau E (2015) Embedded multi-core systems dedicated to dynamic dataflow programs. Signal Processing Systems 80(1):121-136 
64. Zaki GF, Plishker W, Bhattacharyya SS, Fruth F (2017) Implementation, scheduling, and adaptation of partial expansion graphs on multicore platforms. Signal Processing Systems 87(1):107-125 